\title{
. On the dynamic response of flexible dual row retaining walls in dry sand
}

\author{
Srikanth S C Madabhushi \\ Stuart K Haigh
}

\begin{abstract}
Dual row retaining walls can be utilised to form embankment structures for protection against tsunamis or as port structures. The dynamic response of these walls involves a complex interaction between the soil and structural elements which is not well understood. In this paper, for the first time a combination of centrifuge and finite element modelling is used to better understand the mechanical response of the combined wall-soil system. The measured variation of the horizontal stresses leads to bending moment distributions featuring large, singly outward bending or double curvature with significant inward bending of the wall near the ground level. The numerical analyses are used to understand the stress state and highlight the dynamic variations of the vertical effective stress that drive this previously unexplained behaviour. The shear stresses that can develop at the wall-soil interface govern the mechanism by which the vertical effective stresses can vary. Further consideration of the dynamic soil stress state suggests a purer interpretation of the limiting loads in soil-structure interaction problems relative to the traditionally defined dynamic earth pressure coefficients. Combining the altered vertical soil stresses with earth pressure coefficients depending only on the soil friction angle adequately bounds the horizontal stresses that develop in the soil.
\end{abstract}

\section{Introduction}

Modern civil engineers are tasked with designing against increasingly severe natural catastrophes. In particular, recent occurrences of combined earthquakes and tsunamis, such as the 2004 Indian ocean event, the 2011 Tōhoku incident or the 2018 Sulawesi earthquake and tsunami re-established the threat such multihazard events pose. Innovative solutions are required to improve coastal defences worldwide; they must be both robust and efficient to protect populations and be environmentally friendly when deployed over long stretches of coastline. Dual row retaining walls, which feature two parallel rows of sheet pile walls tied at the wall heads with a sand infill, could be one such solution. The design should make economic use of the soil strength and stiffness to enhance the lateral capacity of the system, remaining resilient to both the preceding earthquake and subsequent tsunami. More generally, these wall configurations can also be used as port or embankment structures as they facilitate smaller horizontal footprints. Though these structures are being trialled along the Kochi coastline in Japan, the fundamentals of their behaviour are currently not well understood.

In this paper, a combination of results from dynamic centrifuge testing and numerical analyses focussing on the mechanical response of the system are discussed. The case of a dual row system with flexible walls founded in dry, medium-dense sand is considered. The understanding from this work forms the basis of interpreting the results from more realistic but complex scenarios, such as when the ground is saturated and can liquefy (Madabhushi, 2018). Figure 1 illustrates the dual row retaining wall concept, and highlights how the interaction between the wall and soil controls the overall system response. As the soil shears on horizontal loading the forces transferred will have both horizontal and vertical components. Typically, where two retaining walls are combined as with quay walls etc., the walls are often spaced so as to limit any overlap of potential failure wedges (Ebeling and Morrison, 1992). The impact of closely spaced retaining walls on the soil behaviour and dynamic lateral response has not been widely researched. Potentially, narrower infill's may see proportionally more contribution from the soil to the overall strength and stiffness though a global translation or rotation mode also becomes more likely (Burki and Richards, 1975). 
The dynamic centrifuge tests were conducted to observe the deformation mechanisms in response to large lateral shaking. The experiments utilise traditional instrumentation techniques to measure the structural response of the walls and tie as well as more novel instrumentation such as tactile pressure sheets to better understand the dynamic horizontal soil stresses. Numerical analyses, calibrated against the experiments are then used to provide further insight, particularly in terms of the dynamic variation of the vertical soil stresses.

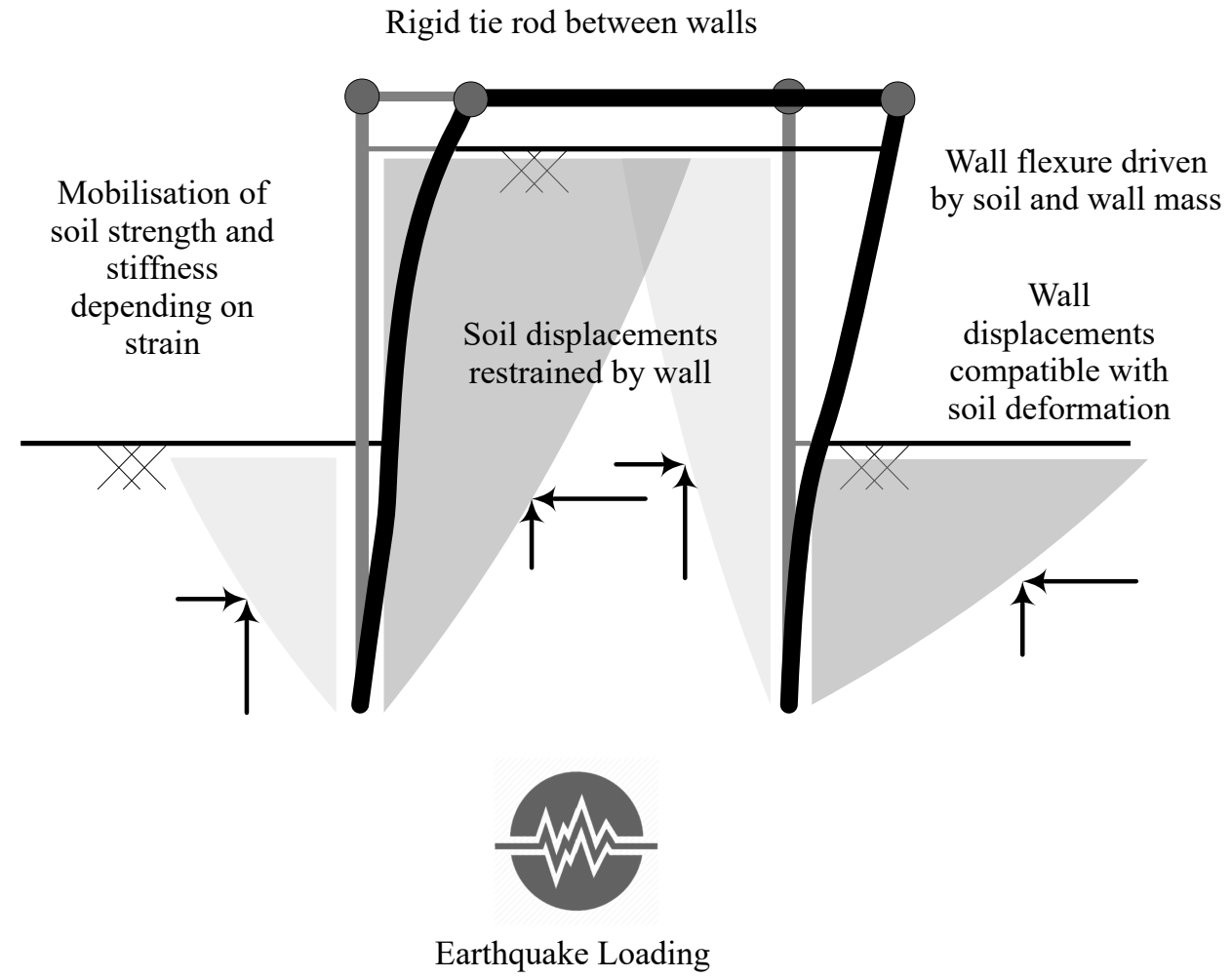

Figure 1: Conceptualization of the dynamic response of a flexible dual row retaining wall system in dry sand

Overall, the use of both investigation techniques in parallel allows the dynamic behaviour to be understood from the system displacements to the stresses in the soil. The system deformations and distributions of the bending moments and soil stresses are discussed at instants of time.

Madabhushi (2018) and Madabhushi and Haigh (2018a) compared the overall dynamic response of dual row wall systems founded in dry and liquefiable soils. The key difference between the responses is the system deformation mode depending on the relative wall to soil stiffness. In dry sands the the systems deformed through flexure or shear but with minimal toe displacement. By contrast, in cases with soil liquefaction the wall toes exhibited outward cyclic ratcheting with rotation about the tie, accumulating displacement throughout the shaking event. Understanding the mechanics of the dual row walls in dry soil is important for their use as port or embankment structures. Further, the mechanics can be contrasted with the cases featuring liquefaction to highlight the regions of soil controlling the wall displacements. Madabhushi (2018) shows that it is primarily the loss of stiffness the soil external to the two walls that alters the deformation mode relative to the dry case, whereas shear induced dilation and suctions between the walls prevent the internal region of soil liquefying. Thus, understanding the dry system mechanics can guide the design of dual row walls in liquefying soils, and particularly help identify the regions of soil where dramatic losses to the strength and stiffness could be critical. Further, the exploration of the dynamic stresses mobilised around the dual row retaining walls in dry sand in this paper motivates a change to the interpretation of dynamic 
design loads more generally.

The design loads that can be generated around retaining structures typically have focussed on the horizontal soil stresses that can develop. These may involve 'elastic' predictions (Scott, 1973; Wood, 1975) for small displacements or rotations. Alternatively, pseudo-static (Okabe, 1924; Mononobe and Matsuo, 1929) or even pseudo-dynamic (Steedman and Zeng, 1990; Choudhury and Nimbalkar, 2006) estimates for the limiting plastic forces for large deformations can be pursued. Predictions of the horizontal stress distributions or total dynamic horizontal force typically utilise an assumed linear, geostatic vertical stress distribution to derive 'dynamic' earth pressure coefficients. These are analogous to those derived for the static cases using a lower bound approach (Rankine, 1856) or an effective upper bound approach (Coulomb, 1776). The drawbacks such design methodologies have on interpreting the soil behaviour during dynamic loading is highlighted in this work. Though these limitations are raised in the context of the complex case of dual row retaining walls, there are broader implications for interpreting dynamic earth pressure coefficients for many cases of dynamic soil-structure interaction problems.

\section{Centrifuge modelling}

A centrifuge is used to accurately replicate the dynamic behaviour of the full size dual row walls when using small scale models. The centrifugal acceleration increases the stresses in the model ensuring that the soil behaviour will be similar to the full scale field case. Schofield (1980) provides details of the centrifuge testing facilities used at the University of Cambridge and the scaling laws used to interpret the results.

\subsection{Model details}

A simplified schematic of the model cross section is shown in fig. 2. The model was subjected to a centrifugal acceleration of $60 \mathrm{~g}$ at $1 / 3$ the dual row wall height, resulting in the equivalent prototype wall system detailed by table 1 . The test is identified as DF666, in reference to the dry sand, relatively flexible wall and retained height, embedment and wall spacing in metres.

A rigid model container with a perspex window facilitated the use of PIV (White et al., 2003). To minimise boundary effects, Duxseal inserts were placed between the soil and the rigid container walls. Coe et al. (1985) and Campbell et al. (1991) have previously discussed the effectiveness of Duxseal in terms of absorbing the stress wave reflections. S28 Hostun sand (Azeiteiro et al., 2017) was used and deposited to an $I_{d} \approx 80-85 \%$ using an automatic spot pluviator (Madabhushi et al., 2006). The container has a width of $200 \mathrm{~mm}$ in the plane strain direction. The model's length to width aspect ratio of 2.15 combined with the polished Perspex front helps reduce the relative influence of the side wall friction on the plane strain behaviour of the model.

The model dual row walls were installed a $1 \mathrm{~g}$, having been placed when the sand level reached $75 \mathrm{~mm}$. Pouring was then continued in and around the wall system. The full earthquake sequence applied to this model has been previously presented by Madabhushi and Haigh (2019). The beneficial effect of an initial, small $(<0.05 \mathrm{~g})$ sine motion in removing any unstable soil arches which may have resulted from the model construction process was shown. In this current paper, the mechanics of the dynamic response of the dual row walls is elucidated by exploring the measured and predicted behaviour during an early cycle of a large $0.35 \mathrm{~g}$ sinusoidal motion, which followed the small non-destructive sine motion in the experiments.

Figure 2 also shows the instrumentation discussed in this paper. The soil accelerations were recorded using piezoelectric accelerometers, whilst lighter, MEMs accelerometers were attached along the wall height. Full bridge strain gauges were used to obtain the flexure of both walls and load cells were used to measure the axial forces in the two tie rods, which featured pinned connections to the two walls. Tactile pressure sensors manufactured by Tekscan were used to obtain the dynamic horizontal effective stress distributions around the right wall. The sheets were laminated and adhered to central portions of the wall, and the remaining areas were covered in acetate to ensure consistent interfacial properties between the walls and soil. Careful calibration and interpretation of the Tekscan output is required, particularly when used with granular materials and dynamic tests (Paikowsky and Hajduk, 1997; Dashti et al., 2012). The processing scheme suggested by Madabhushi and Haigh (2018d) was followed in this work. Madabhushi and Haigh (2018d) also discuss the consistency between the dynamic horizontal stress measurements and the bending 
Table 1: Details of prototype wall system

\begin{tabular}{ll}
\hline Variable & Value \\
\hline Total Wall Height $(m)$ & 12 \\
Embedment Depth $(m)$ & 6 \\
Wall Thickness $(m)$ & 0.18 \\
Bending Stiffness $\left(M N m^{2} / m\right)$ & 34 \\
Tie Length $(m)$ & 6 \\
\hline
\end{tabular}

moments. Unless stated otherwise, results are presented at the prototype scale and the sign convention that leftward displacements are positive is followed.

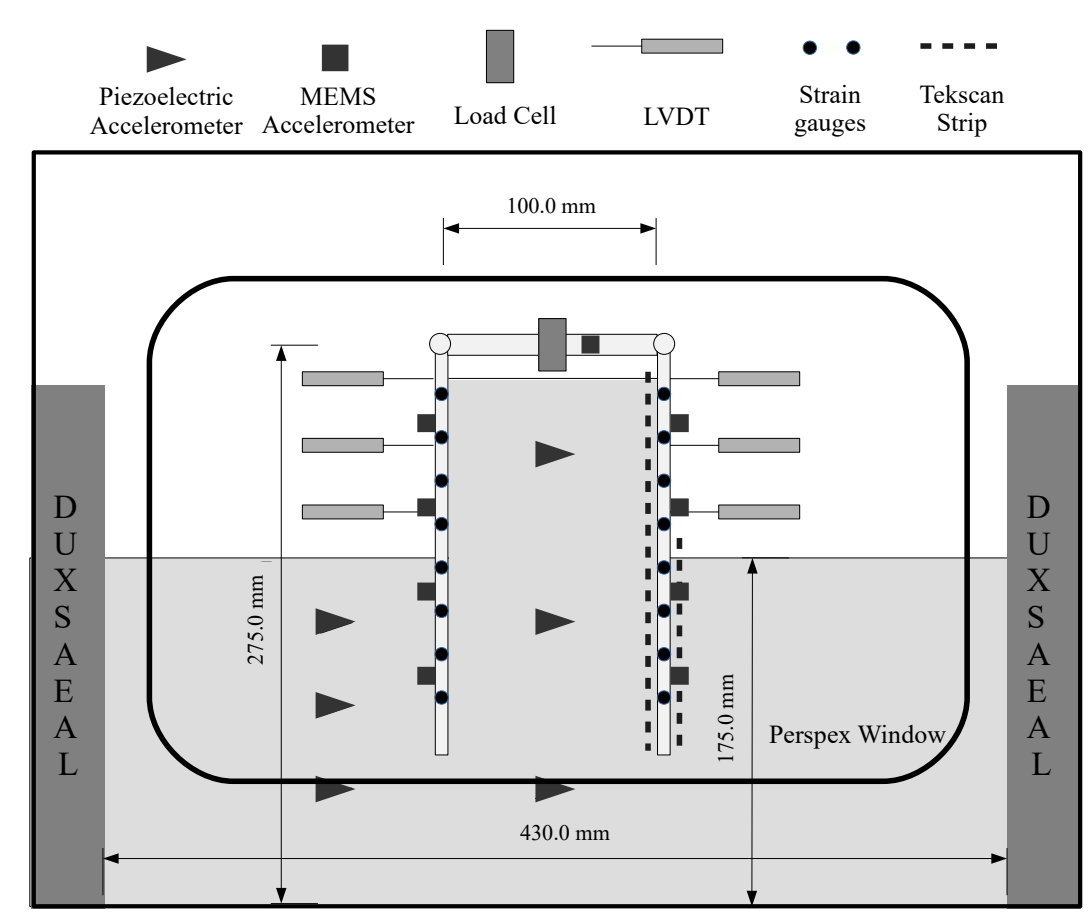

Figure 2: Centrifuge model cross section, model dimensions and instrumentation

\subsection{Experimentally observed dynamic response}

The static equilibrium of DF666, following both swing up and a small sine shake has been discussed in Madabhushi and Haigh (2019) and Madabhushi and Haigh (2018c). In short, both of the flexible walls exhibit double curvature where the outward bending of the top section of the wall is restrained by the tie rod and external soil. The mobilisation of a peak in stresses in this external soil, driven by horizontal equilibrium, also leads to inward curvature of the lower portion of the wall.

On application of a larger sinusoidal ground motion, 'EQ2', the wall bending is found to exhibit two extremes depending on the acceleration direction, resulting in instants of increased outward and/or inward 
bending moment. Madabhushi and Haigh (2019) showed the temporal variations of the dual row system, and, whilst the response showed an overall accumulation of plastic strain, recoverable or 'elastic' cyclic variations are exhibited throughout EQ2. Seeking maxima and minima of the bending moment variation with time reveals that the peak inward bending of one wall is coincident with the peak outward bending of the other and vice versa. The intra-cyclical behaviour, in terms of the wall displacement, bending and inertial accelerations as well as the soil deformations and horizontal stresses are collated to understand the dynamic behaviour of the dual row wall system. Figure 3 shows the behaviour leading to the peak outward bending of the left wall and the peak inward bending of the right wall. Occurring half a cycle later, fig. 4 shows the reversal of this situation. The cycles at the times presented can be considered harmonic in the sense the displacements and accelerations are in anti-phase, which is not always the case during the first and last applied cycles.

The dynamic wall and soil deformations, obtained from the PIV method, are combined with the measured inertial wall accelerations in fig. 3a. The soil displacement vectors and deformed wall shape have been magnified with contours of the magnitude of the soil displacement plotted at the original soil location. The instantaneously applied acceleration to the model base is also indicated. The vertical transmission of the accelerations are both amplified by the soil-wall response and lagged proportional to the system stiffness. The peak rightward displacement at the top of the walls occurs a quarter of a cycle after the peak leftward acceleration is applied at the base. In fig. 3a the left wall is dragged rightwards by the tie rod, and overall the soil forms a collapsing trapezoidal wedge that spans the full extent of the infill. Nevertheless, the tendency of the soil to flow outwards against the left wall is not totally reversed, particularly near the left wall toe, similar to the conceptualised soil wedges in fig. 1. Overall it is confirmed that the wall-soil-wall system is unlike a composite sandwich beam with different flexural stiffnesses; the soil resists deformation through utilisation of its shear strength between inclined - and potentially curved - slipping planes.

Figure 3b shows the measured initial static bending moments, the corresponding horizontal effective stresses around the right the wall as well as the measured tie force between the left and right wall. The same distributions are shown for the four instants depicted in fig. 3a. Concentrating on the right wall, the increased inward bending in the lower portion of the wall is clearly due to the steadily increasing external soil stresses, particularly near the soil surface where there is large relative wall displacement and thus big soil strains. The outward bending at the top of the wall can be increased or decreased relative to the static equilibrium, though the tie rod always experiences tensions greater than the initial value. The internal earth pressures acting on the wall midsection rise as the wall displaces but plateaus at the instant of maximum inward bending of the right wall. Potentially, as the wall moves in the early stages the acceleration of the infill mass will add to the driving stress on the inside of the right wall but as the external soil strains, stiffens and stops permitting displacement (evidenced by the reduced accelerations) the inertial component of the driving stress must reduce. The peak of internal stress below the wall midsection is present before and during the ground motion. It may be attributed to arching of the soil between the walls increasing the mobilised earth pressure coefficient but this phenomenon requires further study.

Figure 4 examines the reversal of the bending scenario described, beginning a quarter of a cycle after fig. 3 ends and culminating with peak outward bending of the right wall and inward bending of the left wall. The main driver for the right wall displacing to the left is the action of the tie from the leftward moving left wall, itself driven by the leftward collapsing infill wedge. The soil adjacent to the right wall, though generally accelerating leftwards, has less tendency to move than the right wall being dragged by the tie and the large left wall movement. This resistance from the soil to the right wall's movement causes outward wall bending. The earth pressures which cause the singly outward bending mechanism (fig. 4b) are revealed and are very different to those associated with the reversed inward and outward bending mechanism seen previously. The external pressures show only modest changes during the cycling and the effective stress distribution increases from the soil surface but does not peak. Internally the effective stresses develop a peak near the ground level and are reduced below this. Having a large peak in stresses acting on the mid section of the wall with reduced external stresses will clearly result in the largest outward bending of the wall. That this peak develops at a higher location than observed when the wall bends in highlights the role of the dynamic wall deformations on the earth pressures generated.

In figs. 3 to 4 the limiting horizontal stresses using the Mononobe-Okabe (Mononobe and Matsuo, 1929; Okabe, 1924) equations with $k_{h}=\mp 0.35 g$ respectively are plotted. These predictions are for the maximum increment of dynamic force due to the inertia of a triangular soil wedge displacing away from or into a rigid 
wall. A triangular distribution of this total force is often assumed to yield equivalent 'dynamic' active or passive distributions. In fig. $4 \mathrm{~b}$, where the accelerations act to increase the passive limit and reduce the active stresses, the infill behaviour is reasonably bounded though this is not the case for fig. 3b where even the predicted active dynamic force is larger than actually occurs. When the system displaces rightwards the largest external stresses are in close agreement with the predicted dynamic passive limit but again the varying mobilisation of stress with depth means the total resisting forces would differ. When the system displaces leftwards the external stresses are reduced but not to the dynamic active limit, meaning in this case the dynamic driving force could be under predicted. Overall, not only is it difficult to envisage prediction of the complex distributions of the dynamically mobilised earth pressures using simplified limit equilibrium methods but the total driving and resisting forces cannot consistently be conservatively captured.

There is a large degree of symmetry in the systems deformations over the half a cycle studied by figs. 3 to 4 . It is also clear why the tie force will cycle at double the driving frequency; in both half cycles the wall system displaces away from and returns to the centre which causes the stretching and compression of the tie as the roles of leading and lagging wall are reversed. Differences in the soil stiffness as the soil stresses cycle towards the active or passive limit states contributes to the dynamic tie forces during the anti-symmetric dynamic loading. Examining the gradients of the cyclic bending moments in figs. 3 to 4 shows that as the shear force at the top of one wall increases the shear force at the top of the other decreases. This can only be attributed to the inertia of the tie rod and load cell. Figure 5 plots the forces that act on the tie inferred from the gradients of the bending moment distributions of each wall and the net force this implies. That the applied shear force from the walls vary in phase is demonstrated. The implied acceleration of the tie rod back-calculated from the net shear forces and tie mass, in terms of magnitude and especially frequency content of the variations, corresponds very well with the directly measured accelerations of the tie rod. This serves to illustrate the internal consistency and quality of the experimental results obtained from a variety of different instrumentation techniques, and the importance of the tie mass with respect to the dynamic tie forces and ultimately flexure of the walls.

\section{$3 \quad$ Numerical modelling}

In order to better understand the dynamic stress states that develop, experiment DF666 was numerically modelled using the the 2D FE Code Swandyne. The general implementation details of this code are described by Chan (1988) and Zienkiewicz et al. (2001). Specific considerations for modelling the plane strain seismic response of the dual row retaining walls have been considered by Madabhushi and Haigh (2018b) and detailed in Madabhushi (2018), and will thus be only briefly summarised here.

\subsection{Model details}

The finite element mesh was constructed from eight noded quadrilateral elements generated using the Abaqus/CAE software, and was unstructured to allow more refinement near the walls, as shown in fig. 6 . The recommendations of Semblat et al. (1999) and Cook et al. (2002) were followed in limiting the maximum element size to one tenth of the propagating shear waves wavelength and the time discretisation to give $10-30$ steps in this travel time. 9 Gauss points were used for the full integration within the soil and structural elements and the $\beta-m$ Newmark scheme (Katona and Zienkiewicz, 1985), with a $\theta$ of 0.5 and $\beta$ coefficients of 0.6 and 0.605 , used for the time stepping.

Prior to applying the dynamic excitation, a 'static run' is completed in Swandyne. The gravitational loading is applied to the mesh to find the geostatic equilibrium solution. This is then applied as the initial stress state to an un-deformed mesh used for the dynamic analysis.

The boundary conditions enforced around the mesh are an important consideration, particularly for dynamic problems. The input displacement was applied to all of the nodes at the base of the mesh. However, in lieu of numerically modelling the rigid side walls and Duxseal inserts the side nodes were restrained vertically and tied to the opposite sides, i.e. they displace equally. The numerical model thus has more ideal boundary conditions; incident p-waves are minimised as each tied 'layer' displaces proportional to the mass and stiffness of the material it encloses. The experiments and numerical analyses may still be validly compared if the Duxseal adequately absorbed the stress reflections in the experiments and the displacements 

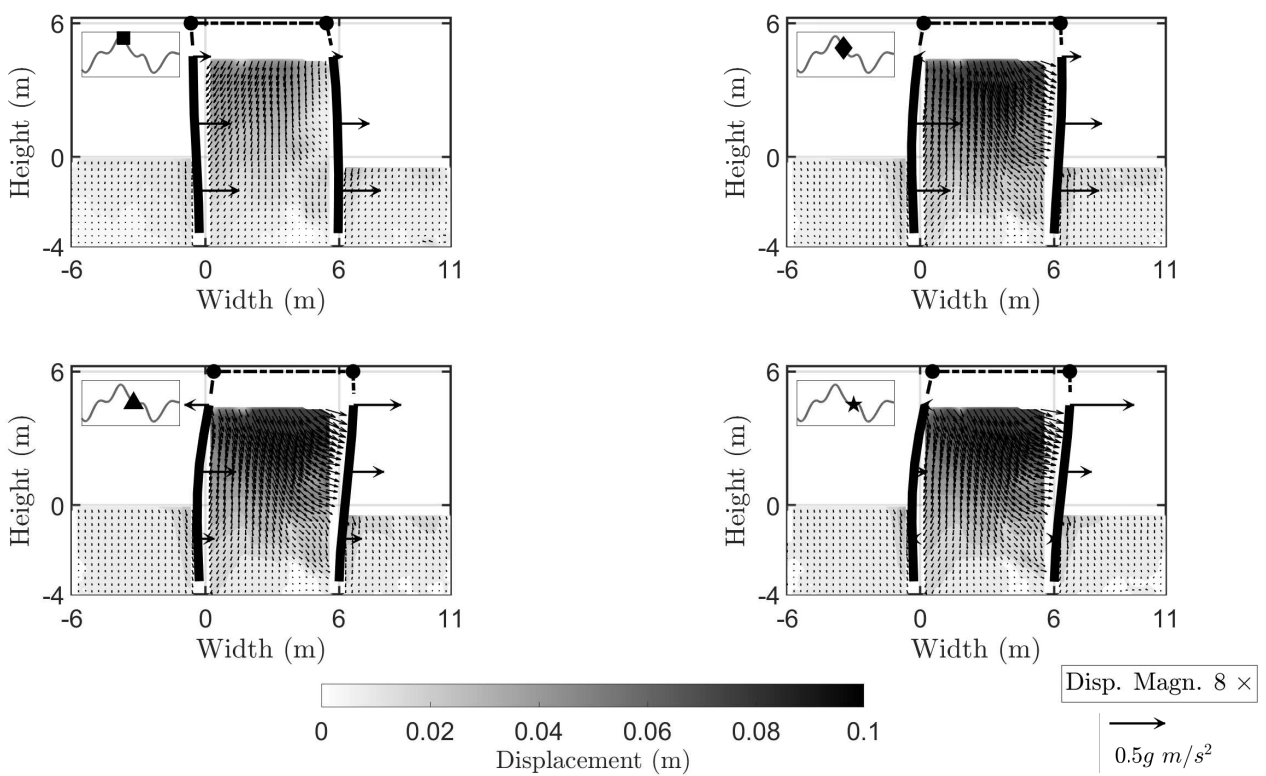

(a) Rightward dynamic displacements and inertial accelerations showing wall flexure and soil shear
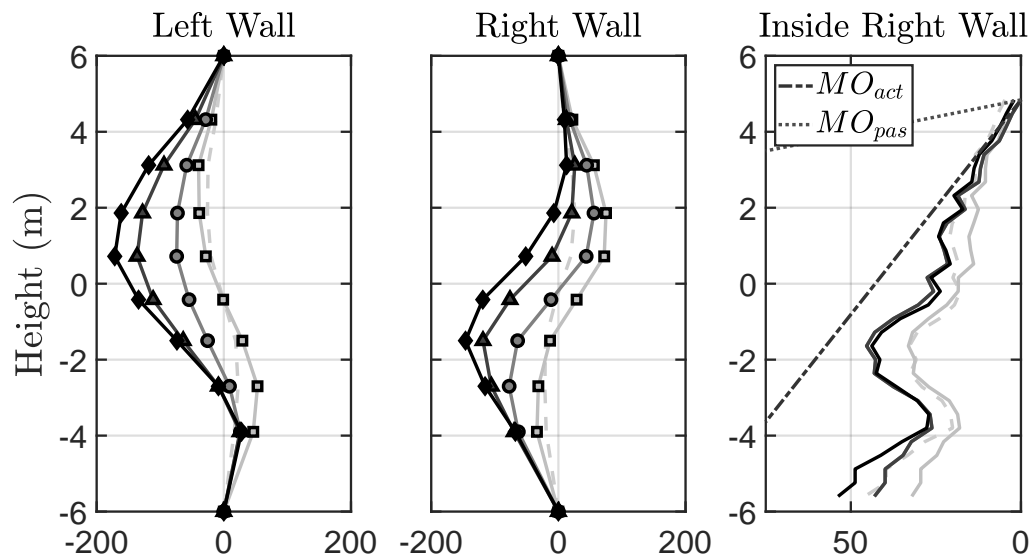

Outside Right Wall

Bending Moment $(\mathrm{kNm} / \mathrm{m})$
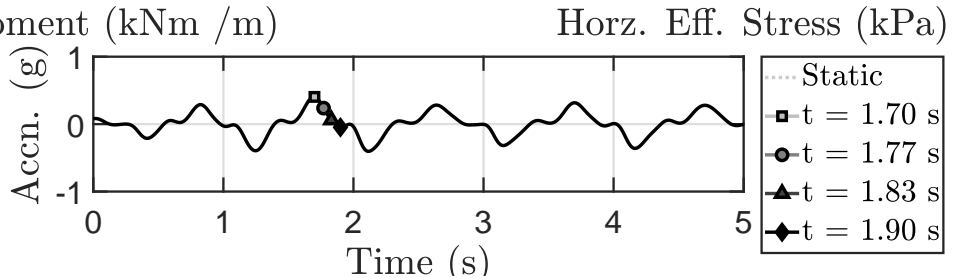

(b) Dynamic bending moments and horizontal effective stresses around the right wall

Figure 3: Instant of dynamic response featuring peak outward bending of left wall and inward bending of right wall 

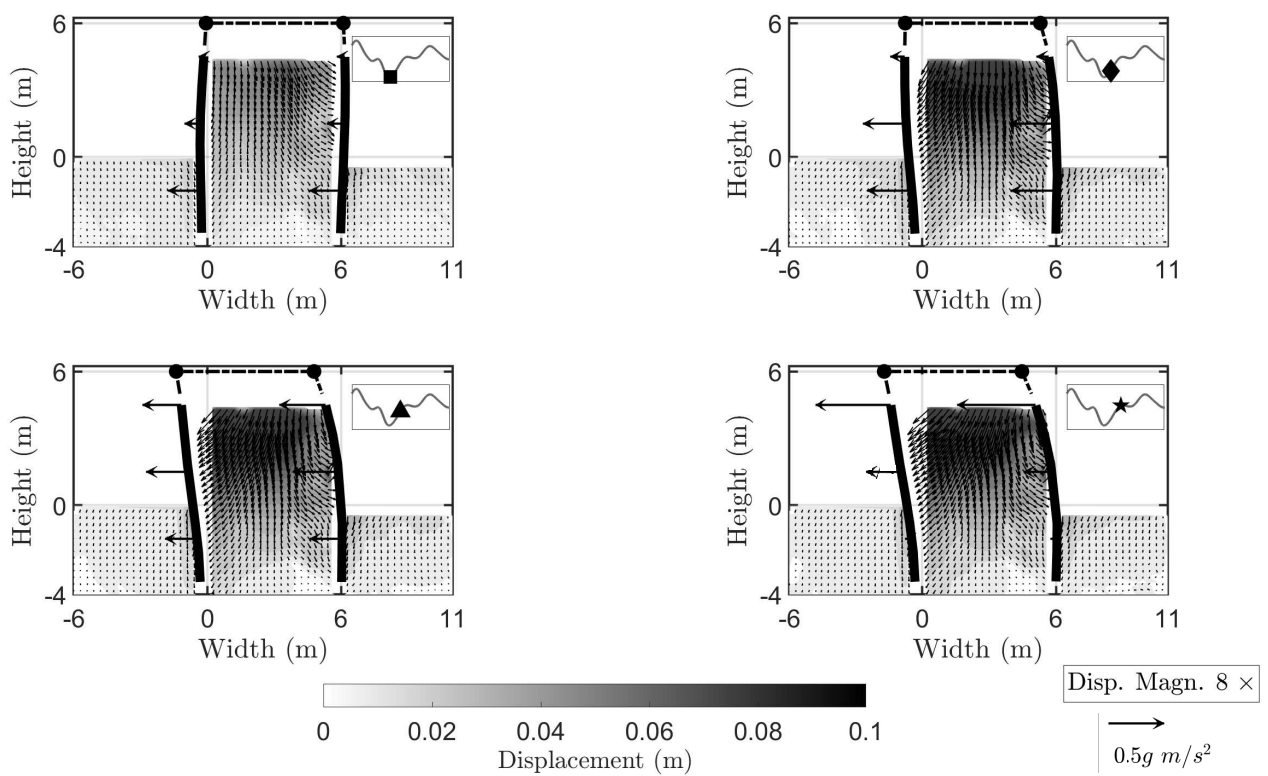

(a) Leftward dynamic displacements and inertial accelerations
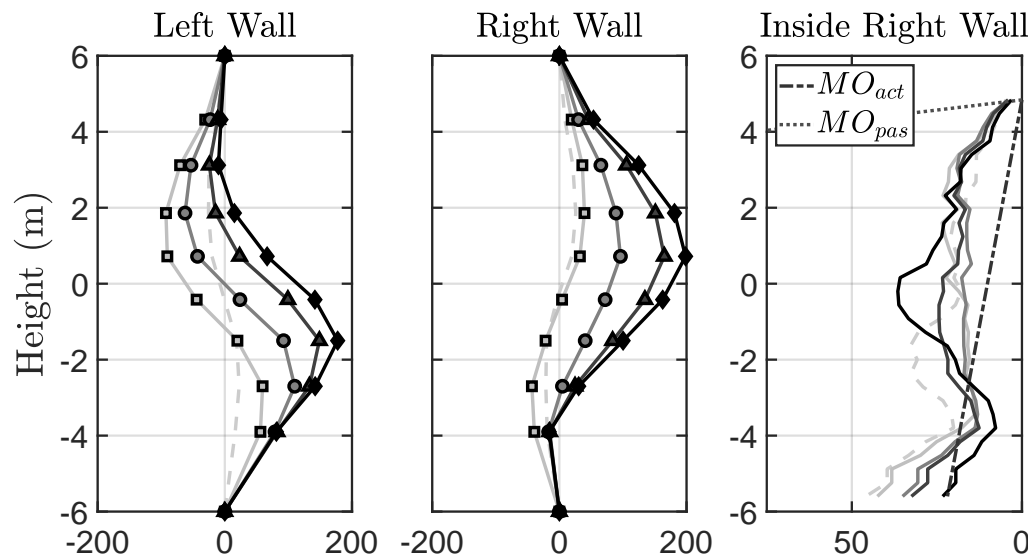

Outside Right Wall

Bending Moment $(\mathrm{kNm} / \mathrm{m})$

Horz. Eff. Stress $(\mathrm{kPa})$
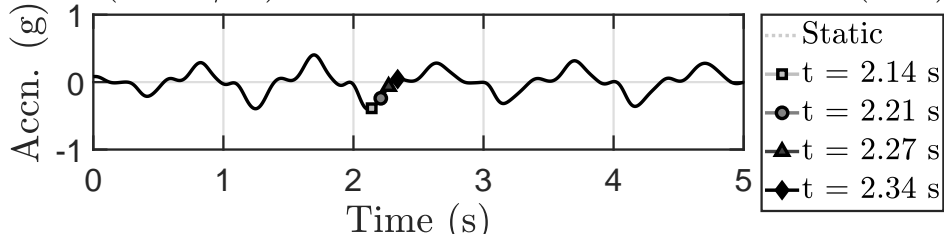

(b) Dynamic bending moments and horizontal effective stress distributions around the right wall

Figure 4: Instant of dynamic response featuring peak inward bending of left wall and outward bending of right wall 

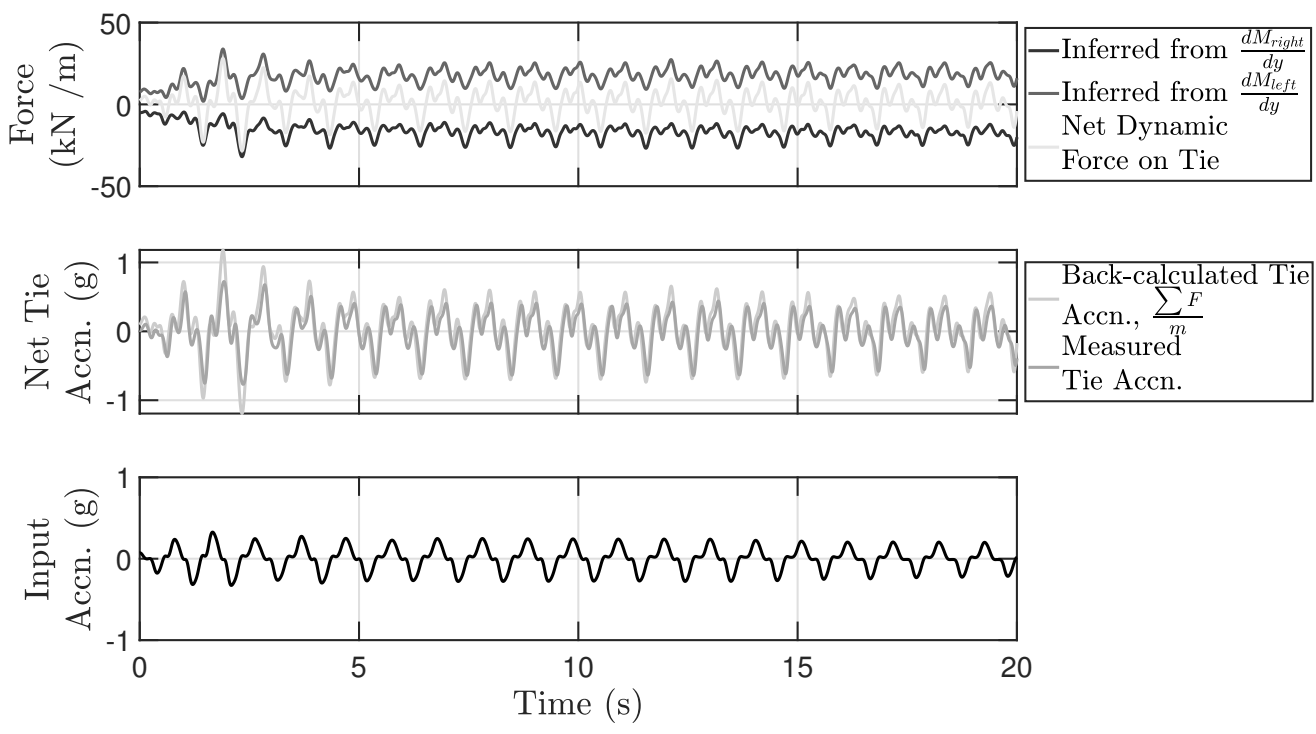

Figure 5: Comparison of the net dynamic tie force, derived from the measured bending moments at the top of the left and right wall, and measured tie acceleration showing the internal consistency of the measurements and importance of the tie mass on the wall flexure

near the boundaries are not significant. That the tests were on medium dense dry sand was beneficial in this respect (Madabhushi and Haigh, 2018b).

The energy imparted to the mesh by the input motion will be partly dissipated by any material hysteresis generated by the constitutive models. However, additional, 'non-physical' damping is often introduced to avoid erroneous oscillations in the numerical solutions. Swandyne includes Rayleigh Damping which requires definition of the proportions of damping from the global mass matrix, $\alpha$ and and initial global stiffness matrix, $\beta$ (Chopra, 2012; Chan, 1988). Kontoe et al. (2011) includes some discussion on the difficulty of choosing Rayleigh damping parameters for dynamic problems where the frequency dependent damping implied is difficult to physically justify. In these analyses, the parameters chosen were broadly based on comparisons with the experimental results - the area enclosed by the experimentally and numerically inferred cyclic stressstrain loops were similar. However, that many different ratios of $\alpha$ and $\beta$ exist, which could give the same overall damping at specific frequencies, should be acknowledged (Madabhushi and Haigh, 2018b).

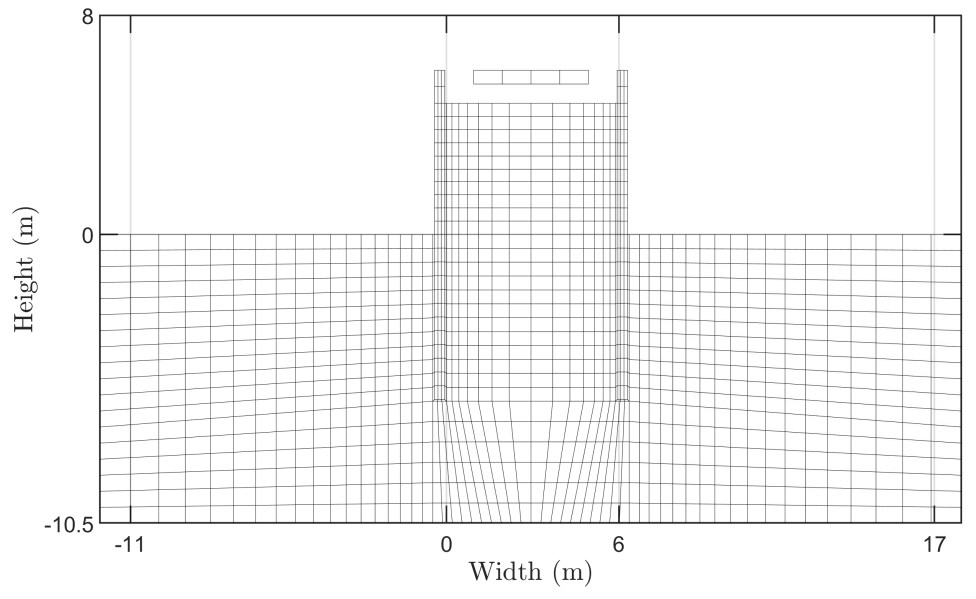

Figure 6: Numerical mesh used for the dual row wall analyses 
Table 2: Parameters used in Numerical Analyses

\begin{tabular}{llll}
\hline & Property / Parameter & Value & Units \\
\hline Structural Elements & Bending Stiffness - Wall & 34 & $\mathrm{MNm}^{2} / \mathrm{m}$ \\
& Axial Stiffness - Tie & 50 & $\mathrm{MN} / \mathrm{m}$ \\
\hline Interface Elements & Young's Modulus (Initial) & 320 & $\mathrm{MPa}$ \\
& Young's Modulus (Dynamic) & 40 & $\mathrm{MPa}$ \\
& Friction Angle & 5.7 & $\circ$ \\
\hline Soil Model & Young's Modulus (Initial) & 320 & $\mathrm{MPa}$ \\
- Mohr Coulomb V & Young's Modulus (Dynamic) & 40 & $\mathrm{MPa}$ \\
& Poisson Ratio & 0.3 & - \\
& Cohesion & 1 & $\mathrm{~Pa}$ \\
& Friction Angle & 33 & $\circ$ \\
& Dilation Angle & 3 & $\circ$ \\
& Reference Stress & 100 & $\mathrm{kPa}$ \\
& Voids Ratio & 0.65 & - \\
& Hardening Parameter & 0 & $\mathrm{~Pa}$ \\
\hline Damping & $\alpha$ & 1.48 & - \\
& $\beta$ & 0.00125 & - \\
\hline
\end{tabular}

A 'floating' tie was used in the 2D Swandyne analysis to simulate the pinned connections; allowing transmission of the vertical and horizontal forces but offering no resistance to moments. Incorporating an elastic tie also allows relative displacement between the walls. As fig. 5 highlighted, being able to account for the inertia of the accelerating tie is also important if the dynamic shear forces and bending moments are to be accurately captured. The measured deflection of the walls from PIV was combined with the recorded forces in the ties to back-calculate an average effective axial stiffness to be used in the numerical analyses (Madabhushi, 2018). This axial stiffness was lower than the theoretical prediction, and was attributed to the physical load cell-tie rod connections. In the numerical analyses the Young's Modulus required for the linear elastic material model was reduced but the corner nodes of the tie were restrained to only lower the axial stiffness but to not reduce the bending stiffness.

A linear elastic model was also used for the elements in the retaining walls, and the overall flexural stiffness was matched with the value in table 1 . At the wall-soil interface slip elements were used. Perfectly rough interfaces with no slip and perfectly smooth interfaces with no shear stress are unrealistic (Clough and Duncan, 1971). Morrison (1995) presents a review of various numerical strategies and interface constitutive models to capture the soil-interface-structure behaviour, whilst Desai et al. (1985) consider the complications added by cyclic behaviour. The slip elements used in this work are a relatively simple implementation, having finite thickness and Coulomb based constitutive model requiring a user specified stiffness and friction angle. The elements are thin eight noded elements with an average aspect ratio of five. The stiffness was guided by the values used for the adjacent soil and a reduced friction angle was used to account for the acetate-soil interface present in the experiments. The influence of the interface friction angle is discussed further in section 3.3.1.

The soil elements were modelled using an implementation based on the Mohr-Coulomb model, which relates the shear stress and normal effective stress at failure to the soil friction angle. Supplying a dilation angle alters the potential function relative to the yield function, which also depends on the plastic strains, meaning the model can exhibit non-associated flow and hardening (Hinton and Owen, 1979). Two stiffness values are defined, firstly whilst solving the geostatic stresses and secondly the initial stiffness mobilised during the dynamic analyses (Chan, 1988). 

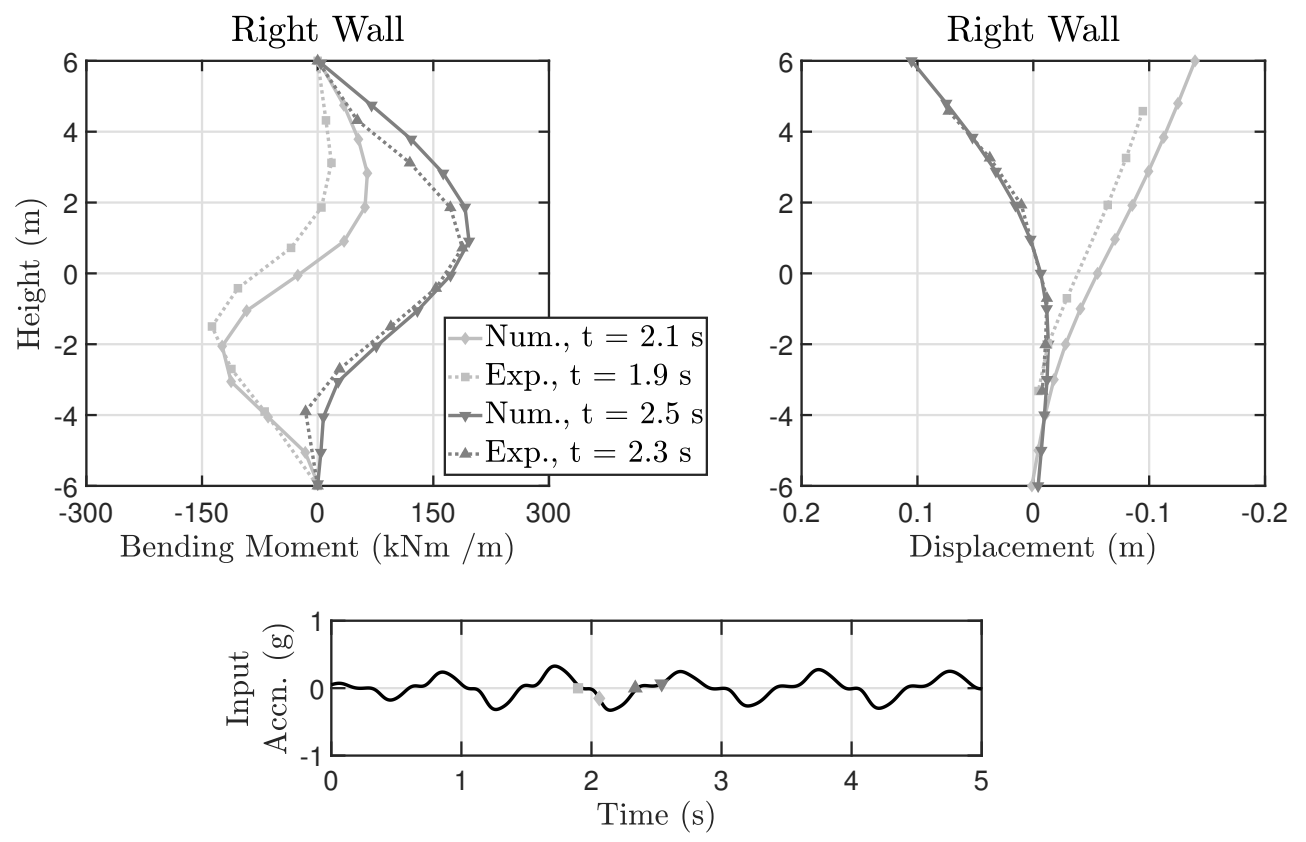

Figure 7: Comparison of measured and predicted dynamic bending moments and displacements to illustrate the overall mechanical response of the dual row walls was accurately captured

\subsection{Parameter Justification}

Madabhushi and Haigh (2018b) presents details of the discussion and justification of the parameter values selected for these analyses and reported in table 2. In the context of this work the numerical analyses are used to further the understanding of the stress state that develops at early instants during the cyclic loading. Therefore, emphasis is placed on capturing the overall mechanical response experimentally observed. In the first instance, the measured and predicted infill soil and wall accelerations can be compared. In judging the accuracy of the vertical propagation of the horizontal accelerations through the model a number of approaches can be taken. Visual comparisons in the time domain are commonly used but the subtleties of the signal transmission can be lost relative to the predominant driving frequency. Conversely, the use of error functions or wavelet transforms to fully examine the progression of different frequency contents with time may also not be desirable if their relative relevance to the overall problem mechanics is unknown. Based on these arguments, Madabhushi and Haigh (2018b) finally present a comparison of the measured and predicted peak amplification envelopes of the wall and soil accelerations. Further, the phase lag of the $1 \mathrm{~Hz}$ component of the vertically propagating accelerations, having a magnitude more than twice as large as the $3 \mathrm{~Hz}$ harmonic and thus being the predominant driving frequency of the input motion, are compared with height. The good agreement from such a comparison suggests the peak dynamic oscillations have been well captured.

The full acceleration time traces can also be used to infer the cyclic shear stress-strain behaviour of the soil. Comparisons between the experimental and numerical loops during the early cycles by Madabhushi and Haigh (2018b) confirm the correspondence of the stresses and strains cyclically mobilised in the soil infill. Ultimately, the measured and predicted forces between the wall and the soil and the associated displacements should match. Figure 7 presents a comparison of the dynamic bending moments and wall displacements for the right wall. The times instants that peak inward and outward bending were experimentally observed are shown alongside the instants with corresponding behaviour in the numerical analyses. The generally observed and predicted behaviours are well matched. The instants at which they occur relative to the base motion are offset due to the differing lag in acceleration propagation numerically predicted. Overall, the additional outputs available from a numerical analyses at these instants can be used with confidence to better understand the dynamic soil stress state. 


\subsection{Numerically predicted dynamic response}

Following fig. 3 the numerical model will be used to study the instant of largest outward bending of the left wall and inward bending of the right wall. The predicted dynamic displacements of the wall and soil in fig. $8 \mathrm{a}$ are consistent with fig. 3a. A measure of the dynamic deviatoric strains, calculated from the differnce in principal normal strains using the small displacement approximations, help to highlight the deformation mechanism of the soil in response to the wall flexure. The collapsing trapezoid of soil between the walls does not move as a rigid block but shows a variation of shear strains. In particular, there are bands of reduced strain between the upper soil that has the tendency to flow left or right against the inside of each wall. The soil below these bands is more isolated from the wall movements and thus strains less.

Figure $8 \mathrm{~b}$ presents the associated bending moments and horizontal stresses from gauss points near the two walls. The corresponding measurements of the dynamic horizontal effective stresses are plotted, with which the numerical predictions are generally in good agreement. Hence, the resulting magnitude and distributions of the outward bending in the left wall and reversed curvature in the right wall are also well matched. Figure 8b additionally shows limit state distributions of horizontal stress, calculated assuming linear vertical stress distributions multiplied with the active and passive Mononobe Okabe coefficients with $k_{h}= \pm 0.35$ for the right and left wall respectively. Comparison between the stresses mobilised under this large dynamic loading and the limiting values of stress with depth are not favourable for most of the soil at this instant. Again, the predictions are not good bounds for the smallest or largest stresses that can be experienced and the variations of horizontal stresses with depth are not particularly linear.

To further understand the soil stress state, the numerical results are used to construct Mohr's circles of stress around the walls at various depths before the shaking and at the instant shown in fig. 8. The stress states for the static equilibrium are relatively straightforwardly interpreted. The stresses around the walls are symmetric with the infill soil generally reaching the active limit. Outside the walls the soil near the surface mobilises a passive peak near the surface - driving the reversal of curvature in terms of bending (fig. 3b) - and at greater depth the horizontal stresses mobilised are reduced. In all cases the anti-symmetric shear stresses mobilised are relatively small as necessitated by the reduced interfacial strength prescribed.

The dynamic stress state shown by fig. 9 reveals a number of interesting trends. In addition to the variation of the horizontal effective stresses, it is clear that there can be significant dynamic variation of the vertical effective stresses - particularly within the infill. As the system displaces rightwards the soil outside the left wall is unloaded towards or fully reaches the active limit. Inside the left wall the statically active soil experiences a dramatic reduction of both the horizontal and vertical stresses. The soil inside the right wall starts at the same active state but the dynamic horizontal and vertical stresses are generally both increased. For the depths shown, the soil is at the active limit and thus the vertical effective stresses exhibit a large increase. Outside the right wall the Mohr's circles plotted reach the passive limit state with the vertical stresses reduced relative to the static equilibrium. That the envelopes based on the critical state friction angle are never violated will be returned to in section 4 .

Deviation of the vertical effective stresses in the soil neighbouring a retaining system or other buried system away from the geostatic, as a result of the imposed kinematic constraint and interfacial shear stresses, is known as soil arching. Typically, analytical solutions for arching have shown that the static vertical effective stresses are reduced below the geostatic values on the active side of a retaining wall (Michalowski and Park, 2003; Paik and Salgado, 2003; Khosravi et al., 2016). The resulting non-linear vertical effective stresses are reduced as a function of the soil - wall interface friction. Figure 8 shows that under dynamic loading the vertical effective stresses can both increase and decrease around the dual row retaining walls.

The potential for significant variation of the vertical effective stresses below a dual row retaining wall has been previously raised by Kitajima et al. (1981), based on static lateral load tests on similar structures. A schematic illustration of the mechanisms that were suggested is presented in fig. 10a and shows the potential for increased vertical effective stresses. More recently, Madabhushi and Haigh (2018b) showed that, under dynamic loading, the vertical effective stresses can also similarly vary within the infill based on the comparison between the numerically predicted stress state and experimental measurements. In fig. 10b the distributions of vertical effective stress leading to the instant shown in fig. 8 are plotted. Figure 10b shows over the course of a quarter of a cycle how the vertical effective stresses inside the right wall are increased in time with a reduction of the stresses inside the left wall. Section 3.3.1 discuss plausible mechanisms for these dynamic vertical effective stress variations between smooth walls and is thus distinctly different to previous studies 


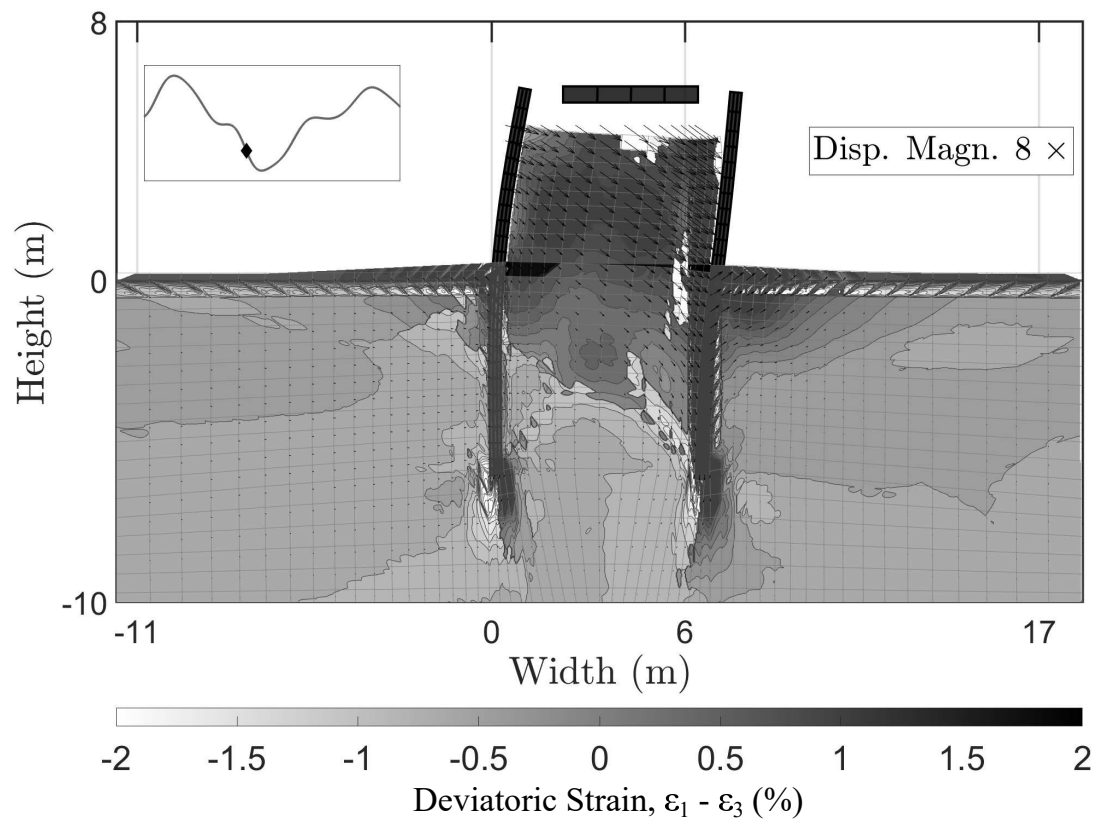

(a) Dynamic displacements and deviatoric shear strains showing wall flexure leading to greater shearing of the soil near the internal and externals soil surfaces
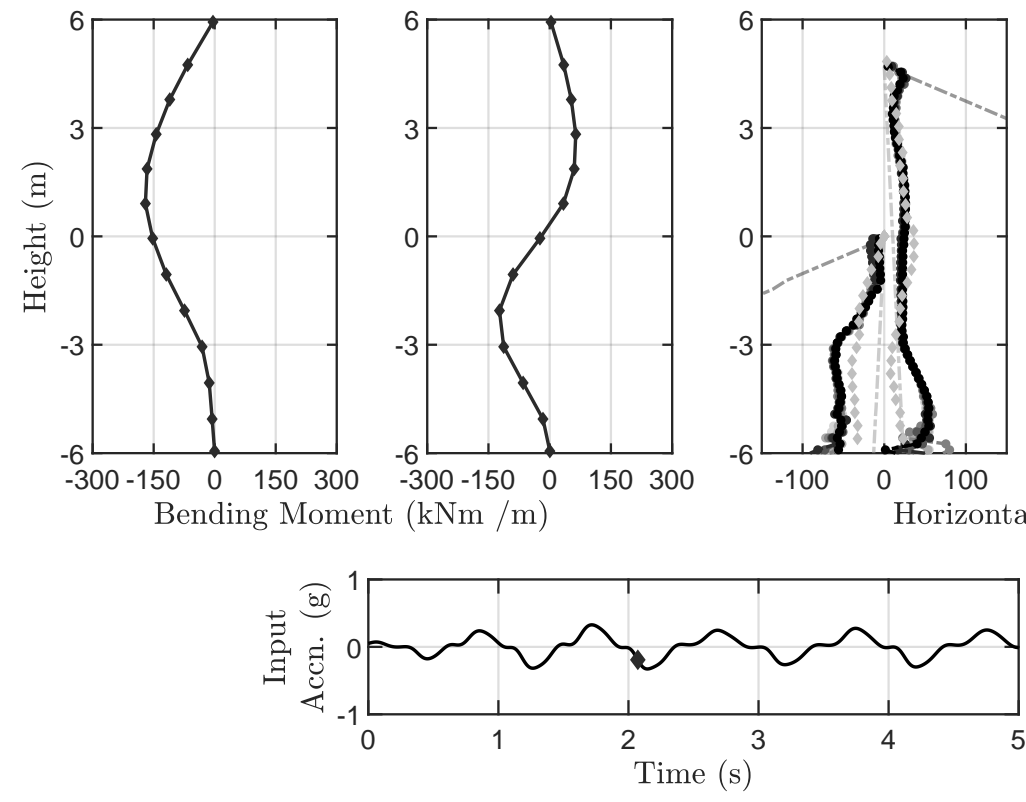
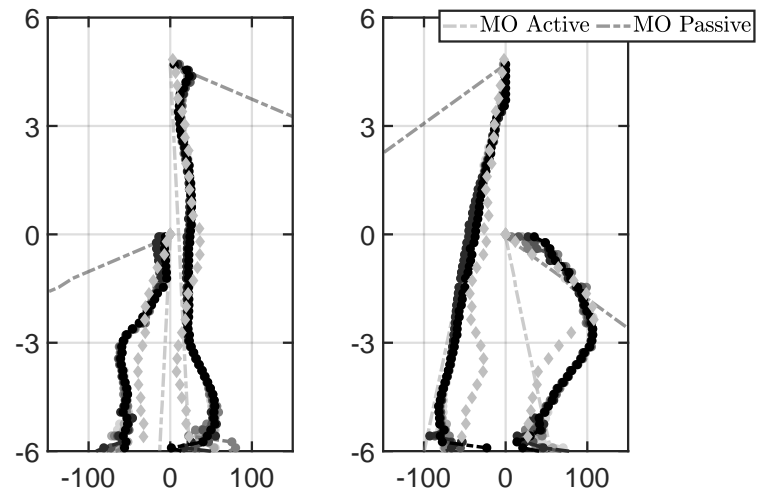

$\rightarrow$-Gauss Point output closest to wall $\rightarrow-$ Gauss Point output at $0.3 \mathrm{~m}$ from wall - Corresponding Exp. Measurement

(b) Bending moments and measured and predicted horizontal effective stresses, both of which can violate traditionally used pseudo-static limiting stress predictions

Figure 8: Numerically predicted dynamic response at $t=2.07 \mathrm{~s}$ 

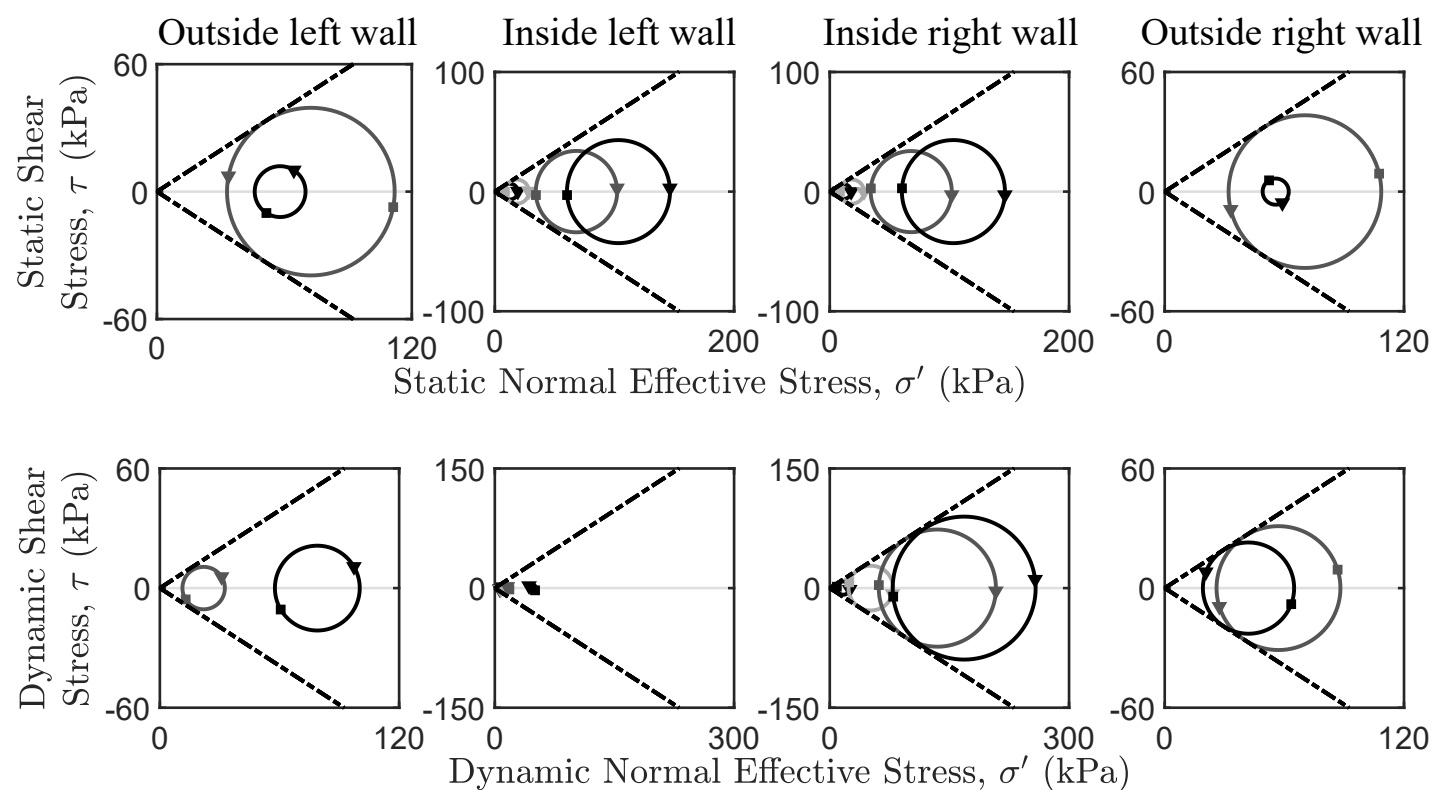

Dynamic Normal Effective Stress, $\sigma^{\prime}(\mathrm{kPa})$

$$
-\mathrm{h}=3 \mathrm{~m}-\mathrm{h}=1.5 \mathrm{~m}-\mathrm{h}=-1.5 \mathrm{~m}-\mathrm{h}=-4 \mathrm{~m}
$$

- Horizontal Principal Stress

- Vertical Principal Stress

Figure 9: Mohr's circles of stress from static equilibrium and $t=2.07 \mathrm{~s}$. Significant variation of the major and minor principal stresses reveals significant variation of both the horizontal and vertical effective stresses during the dynamic loading

on soil arching altering the static vertical effective stresses. Outside both the left and right wall a slight reduction of the vertical effective stress can also be discerned, particularly at the soil nearer the surface, and is returned to in section 4 .

\subsubsection{Rocking Mechanics}

The vertical effective stress oscillations of the infill seen in fig. 10b may be explained by a rocking tendency of the dual row walls. Figure 11a shows a schematic illustration of the rocking mechanisms and the dependence on the friction at the wall-soil interface. The contrast showcased by fig. 11a is analogous to whether 'plugging' can occur within a pile, though in either case vertical stress oscillations can occur.

In the first case, where the system has a tendency to rock under dynamic loading and the walls are rough they will apply shear stresses to the infill soil. The left wall will effectively pick up the infill soil whilst the right will drag it down. This will cause decreased and increased vertical stresses below these sides respectively, which overall supply a resisting moment to the system rotation. On the other hand, if the walls are very smooth they will be unable to apply any shear stresses to the infill soil. As the base shaking is applied and horizontal shear stresses propagate vertically complementary shear stresses must be generated perpendicularly. The inability of the wall-soil interface to provide these shear stresses could lead to a net overturning moment on each 'layer' of soil between the walls. Again, a variation of vertical stresses will be generated in reaction to the moment from this soil region which will also oscillate as the applied input motion oscillates. As the system or soil rocks in a given direction, the vertical stresses on that side are increased whilst the soil on the opposite side is effectively unloaded. The vertical stress distribution below the rocking soil region may depend on the spacing of the two walls, however effects due to reduced complementary shear stresses have been shown even across the whole width of a centrifuge model container (Teymur and Madabhushi, 2003). Figure 11b presents the numerically predicted variation of the shear stresses on the $x-y$ planes within the infill during half a cycle of applied shaking. The shear stresses mobilised within the soil infill decrease with height as did the vertical stress variations observed in fig. 10b. Further, across the 


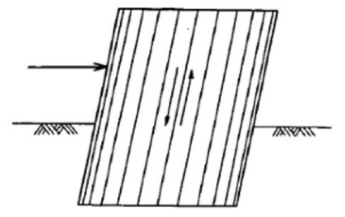

(a) $x+\cdots$
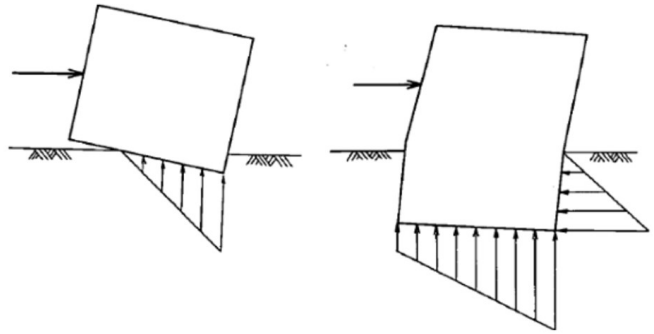

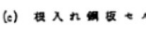

(a) Horizontal load on a cofferdam, following Kitajima et al. (1981), illustrating potential mechanisms for varying vertical effective stress
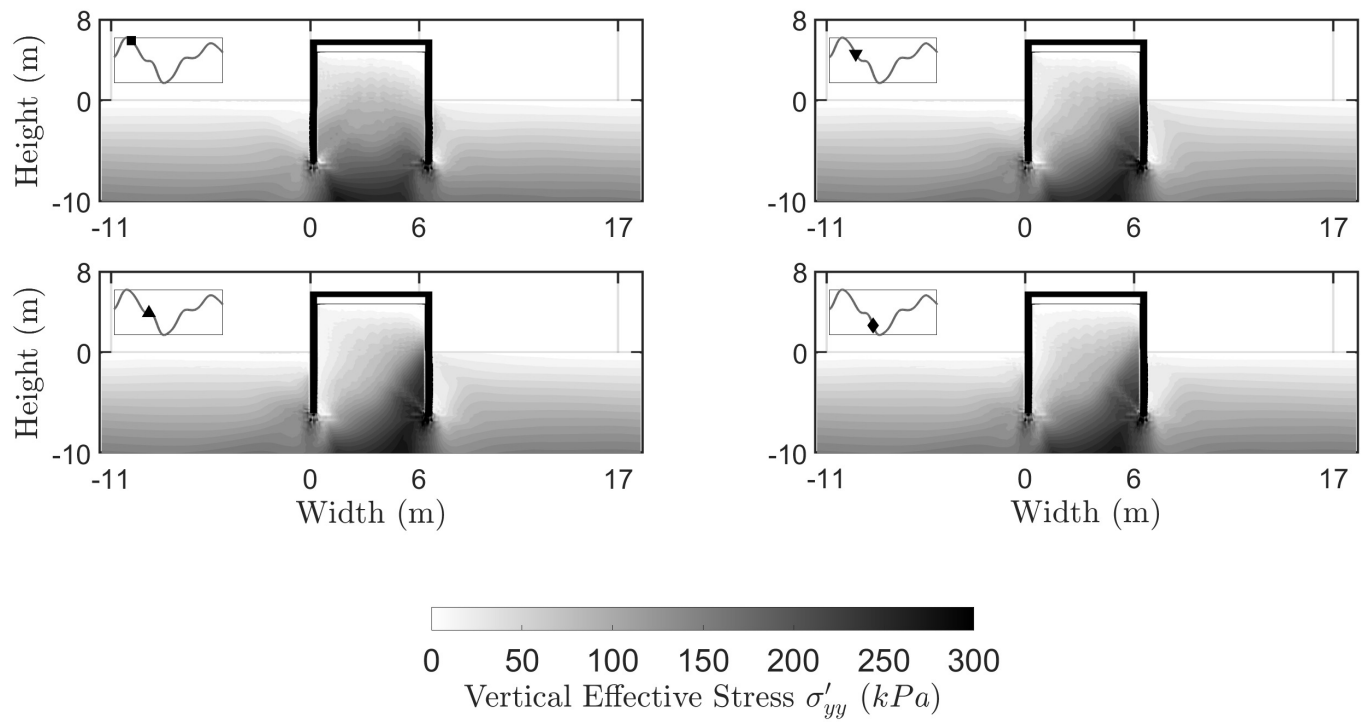

(b) Dynamic variation of vertical stresses numerically predicted beneath and between the dual row retaining walls

Figure 10: Vertical stress variations for dual row walls

half cycle plotted the distribution of shear stresses are shown to clearly reverse. Whilst large shear stresses can develop a few metres inside either wall, the reduction of shear stress toward the wall faces are clear. Overall, the 'rocking infill' mechanism in fig. 11a appears predominantly responsible for the vertical stress variations predicted in these analyses.

To investigate the influence of the wall roughness the specified strength was varied between an interface friction angle, $\delta$ of $0-33^{\circ}$. Figure 12 presents the vertical stress distributions and shear stresses in the infill at the instant of peak outward bending of the left wall for the cases analysed. Figure 12a reveals that though in all cases the vertical effective stresses imply an overall rocking, the magnitude of variation diminishes as the interface strength between the soil and the wall is increased. In fig. 12b the shear stresses across the infill are also seen to vary as a result of changing the interface friction - highlighting that the influence of the wall roughness extends across the width of the soil between the walls. Figure 12b implies that the transition between a rocking infill and overall rocking system is not gradual. The first three cases are fairly similar; the major difference being the increasing shear stress supported towards the inside of the two walls and the reduced overturning moment implied by the asymmetric distributions. In the final case however the mechanics are more significantly altered, with the entire width and height of the infill being sheared in the 
opposite direction which helps resist the system overturning.

In closing, fig. 11a illustrates two mechanisms by which the vertical effective stresses may vary in the soil beneath the dual row walls; a 'rocking system' mechanism if the walls are rough or a 'rocking infill' if they are smooth. Both of these mechanisms are plausible within the wall height, and fig. 10b shows there is a cumulative effect resulting in the greater vertical stress variation with depth. Figure 10b also shows a concentration of vertical stress beneath the dual row walls at all instants of the dynamic shaking. The proximity of the rigid base boundary, based on the experimentally tested dimensions, may be increasing the soil stresses experienced in this region. However, the tendency for rocking arises from the dual row walls and infill with the soil region below reacting to these changes. Thus, a deeper soil layer may exhibit a more diffuse stress bulb but the overall mechanics of the soil, particularly between the walls, would not be expected to change.

\section{Broader implications for the use of dynamic earth pressure co- efficients}

Dynamic earth pressure coefficients, similar to those used for limit equilibrium design for static conditions, are typically derived by obtaining an estimate of the total dynamic force (from upper bound style solutions (Okabe, 1924; Mononobe and Matsuo, 1929)) and dividing this by an assumed, linear vertical stress distribution to get a linear distribution of horizontal stresses. A common criticism of these methods has been the assumption of full mobilisation of the soil strength and assuming the horizontal stresses are linearly distributed. As originally proposed by Dubrova (1963) with subsequent modifications summarised by Chang and Chen (1990), non-linear distributions of horizontal effective stresses can be predicted by prescribing partial mobilisation of the soil strength along the wall height. Nevertheless, such methods haved also typically employed geostatic vertical stresses. At first glance, acknowledging the dynamic variation of the vertical effective stresses, as shown to occur both inside and outside the dual row walls, raises an additional complexity to the horizontal stresses that may act on a buried structure.

However, fig. 9 also hints that acknowledging this variation of vertical stresses could simplify the interpretation of the limiting dynamic soil behaviour. Figure 13 explores the numerically predicted dynamic response of the soil. Half of the dual row system, which shares similarities with a single tied retaining wall, is considered with the response of the soil at two locations shown in fig. 13a investigated. The kinematics of wall movements shearing the neighbouring soil imply horizontal and vertical components of soil displacement. If the soil in these regions is accelerating both the horizontal and vertical stresses can be altered.

Figure 13b shows the time varying accelerations and effective stresses in the horizontal and vertical direction predicted at the marked locations. In response to the horizontally applied base shaking the soil inside and outside the right wall is horizontally accelerated. A vertical component of acceleration is also generated at both locations, with peak values $\approx 25 \%$ of the peak horizontal accelerations. However, a vertical acceleration of $0.1 \mathrm{~g}$ can only induce vertical stress changes $10 \%$ of the geostatic values, whereas dynamic increases of up to $100 \%$ were predicted. Between the two walls, the redistribution of vertical soil stresses needed to equilibrate the rocking soil mass (fig. 11a) at every elevation is thus predominantly responsible for the dynamic vertical stress oscillations. Outside the wall, the vertical accelerations kinematically imposed by the diagonal soil shearing can again not fully account for the predicted vertical stress variations. The potential of the external wall-soil interface to induce vertical stress variations due to the interface roughness or conversely a lack of complementary shear stress with the neighbouring soil requires further research.

The final plot in fig. 13b shows the ratio of the horizontal to vertical effective stress mobilised with time. The values will be close to the ratio of principal stresses as the predicted shear stresses were very small (in fact, from the Mohr circle geometries in fig. 9 even a shear stress of $20 \%$ of the maximum shear stress would imply a $2 \%$ change in the maximum principal stress). The ratios inside and outside the right wall are broadly cycling in anti-phase, with the infill tending to an active state whilst the external soil is driven to a passive state. That these states can be partly reversed with time - the external soil being unloaded towards an active state with the infill showing spikes of mobilised passive strength - highlights the complexity of the dynamic design of retaining systems. As the cyclic loading reverses direction instants of maximum structural demand or deflection in either direction should be considered (Kramer, 1996).

The bounds that the stress ratios fluctuate between are also very interesting. Figure 13b presents the 


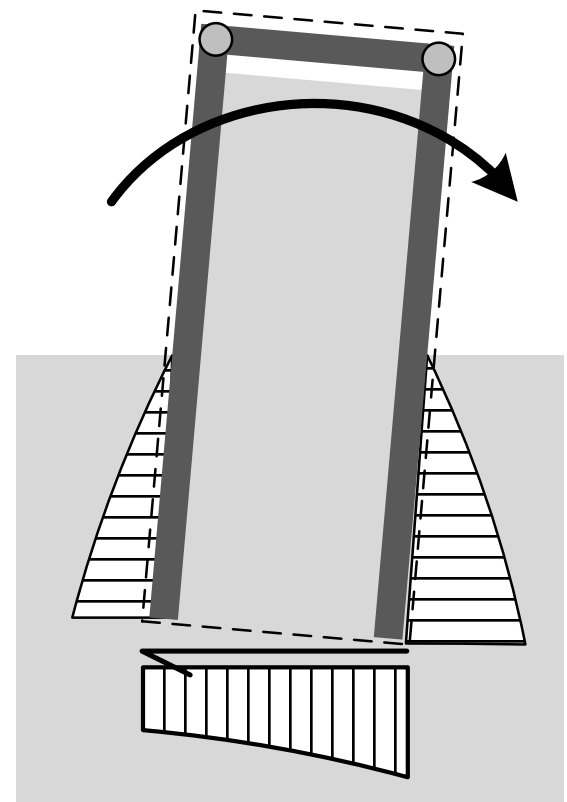

'Rocking System'

Requires Friction between

walls and soil

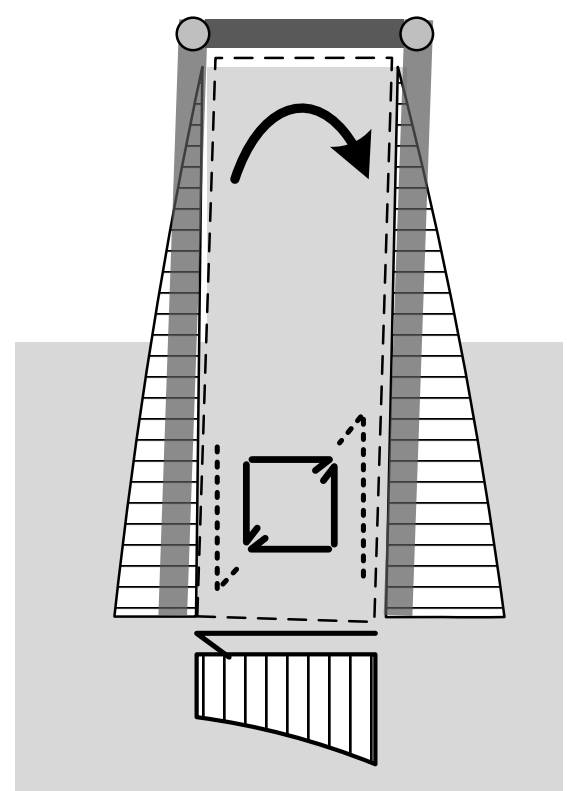

'Rocking Infill'

Lack of complementary shear at the wall-soil interface

(a) Potential rocking mechanisms of wall-soil system or infill soil depending on the availability of complementary shear stresses at the wall-soil interface

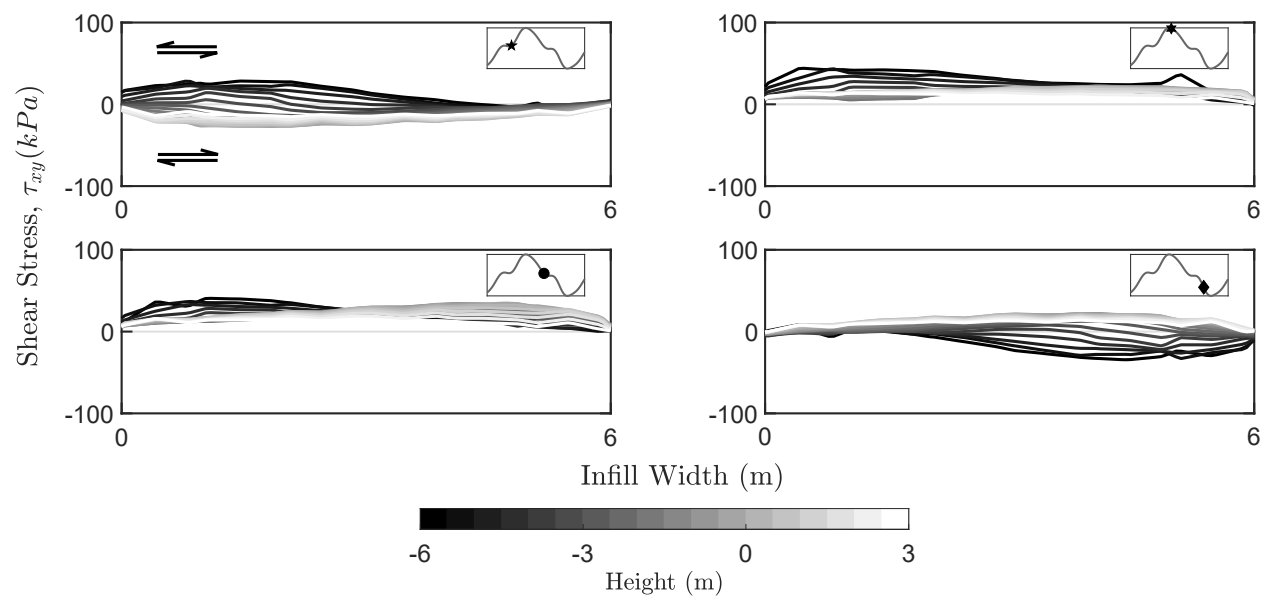

(b) Cyclic variation of shear stresses within infill

Figure 11: Proposed rocking mechanics of the dual row wall system to justify the dynamic vertical effective stresses 

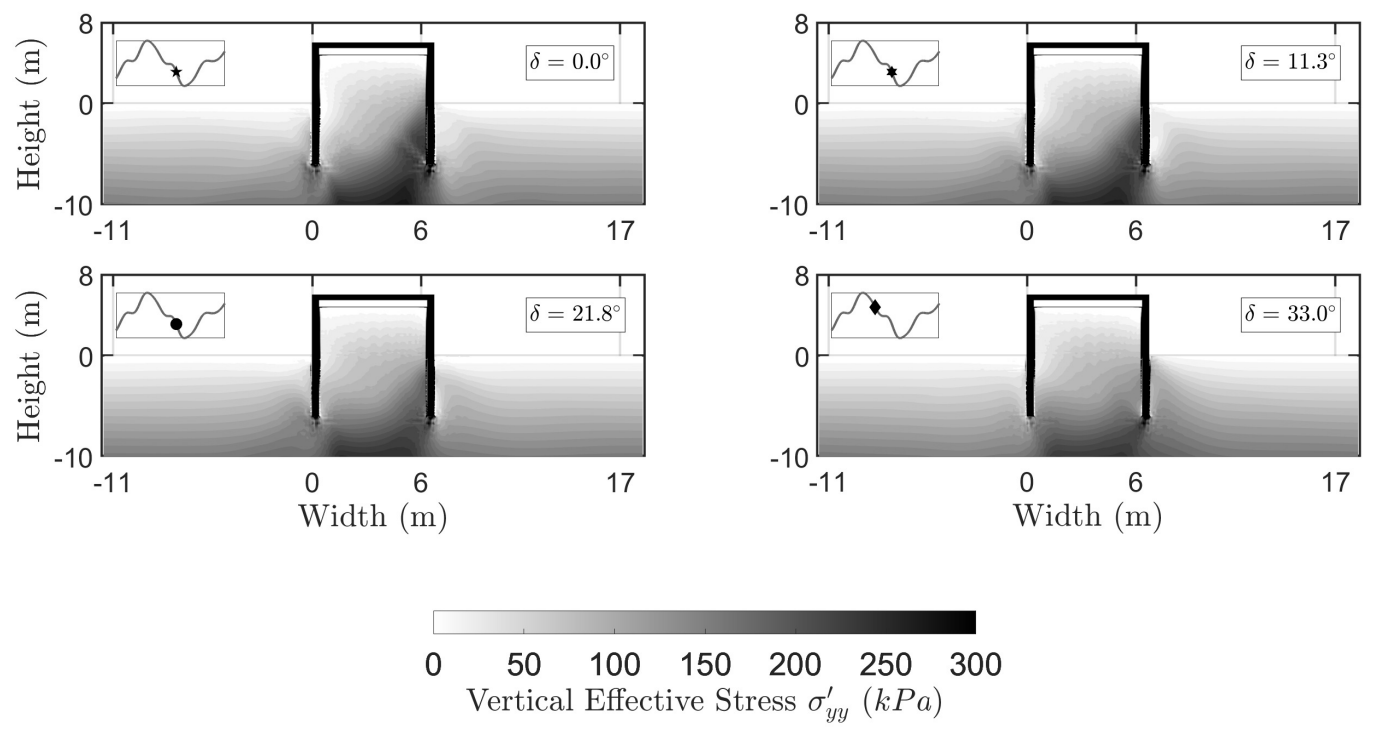

(a) Dynamic variation of vertical stresses

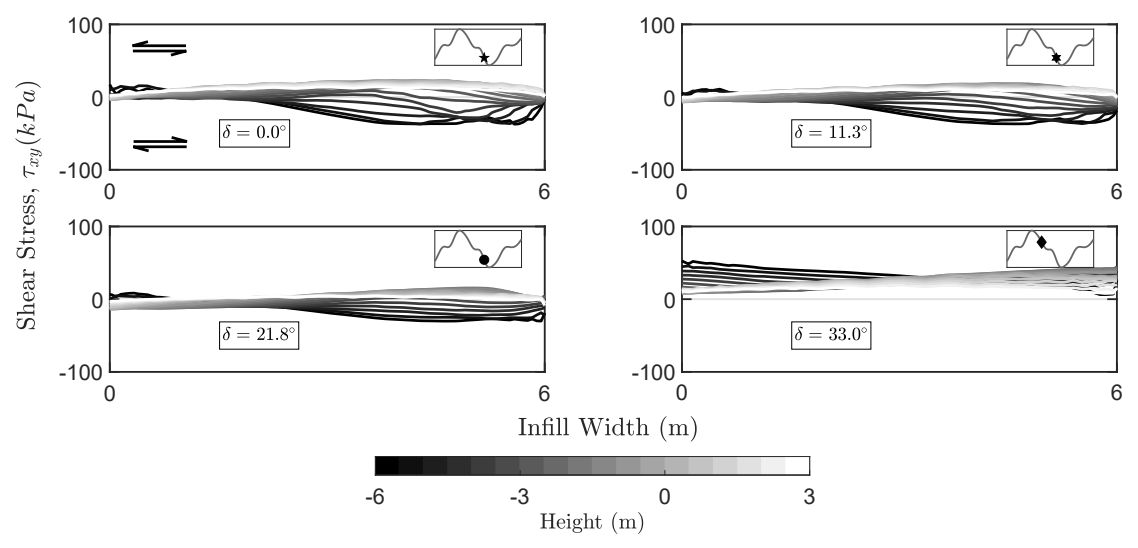

(b) Cyclic variation of shear stresses within infill

Figure 12: Investigating the influence of interface friction on the rocking mechanics confirms dynamic vertical stress variations for all cases

Mononobe-Okabe coefficients, calculated as described previously, with a positive applied acceleration and the so called 'static' earth pressure coefficients. The values of $K_{A} \& K_{P}$ are calculated using only the soils' critical state friction angle (Rankine, 1856). The coefficients derived from predicting the limiting dynamic horizontal force with linear, geostatic vertical forces are clearly not respected. On the other hand, it is evident that the Rankine (1856) coefficients are sufficient to describe the limit state that the soil on either side of the wall can reach. Whilst the mobilisation of the soil strength can vary with time, the Mohr circles of fig. 9 and the results in fig. 13b show that the soil strength under this dynamic loading (and thus induced strain) is limited purely by the critical state friction angle.

In other words, the traditionally defined and used dynamic earth pressure coefficients may be needlessly 
obfuscating the soil behaviour during the earthquake. If greater dynamic active loads or reduced passive resistance are found around a buried system, it is more logical to attribute to this to altered vertical stresses rather than a change of the limiting earth pressure coefficients that can be generated. From a design perspective, predictions of dynamic stress states can focus on the variations of vertical stress (perhaps from rocking mechanisms or from the component of vertical acceleration of the soil mass). The corresponding mobilisation of horizontal stresses required to equilibrate the inertial loads are then required, but the key point is that these are limited by the values of the Rankine (1856) earth pressure coefficients. This conclusion is valid for a granular material where the macroscopic strength can be described by a friction angle.

\section{Conclusions}

A combination of centrifuge modelling and finite element modelling has been used to investigate the dynamic response of a flexible dual row retaining wall system. The results from the centrifuge test revealed the mechanical response of the soil and structural system, with the instrumentation focussing on the wall and soil displacements, the horizontal soil stresses and the resulting wall bending. The dynamic variations of horizontal stress that lead to the greatest singly outward bending of the walls and the distribution half a cycle later, which results in double curvature in the wall with a peak inward bending moment, are shown. The tendency of the soil to shear and thus displace horizontally and vertically in response to the horizontal flexure of the walls was also demonstrated.

A finite element analysis of the centrifuge test was performed to better understand the soil stress state. The model was calibrated against the centrifuge data in a mechanical sense, with the confidence in the overall body forces and displacements predicted increased by comparison with the measured wall bending and displacements. The predicted horizontal response of the system largely confirmed the experimental observations, but the variations in the vertical direction prompted further study. Dynamic variations of the vertical effective stress were predicted, with oscillations in the soil between the two walls as well as outside it. Plausible mechanisms based on the system rocking or from only the soil rocking are presented which

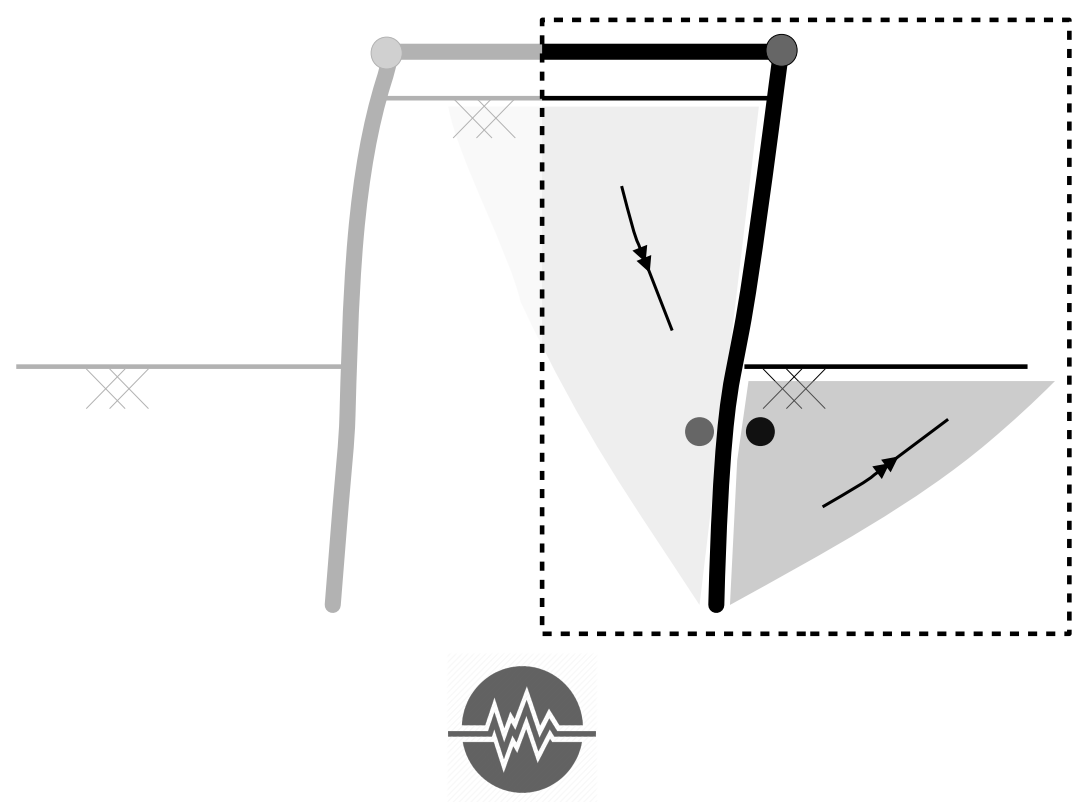

(a) Movement of soil around a single 'tied' wall in the dual row wall system 

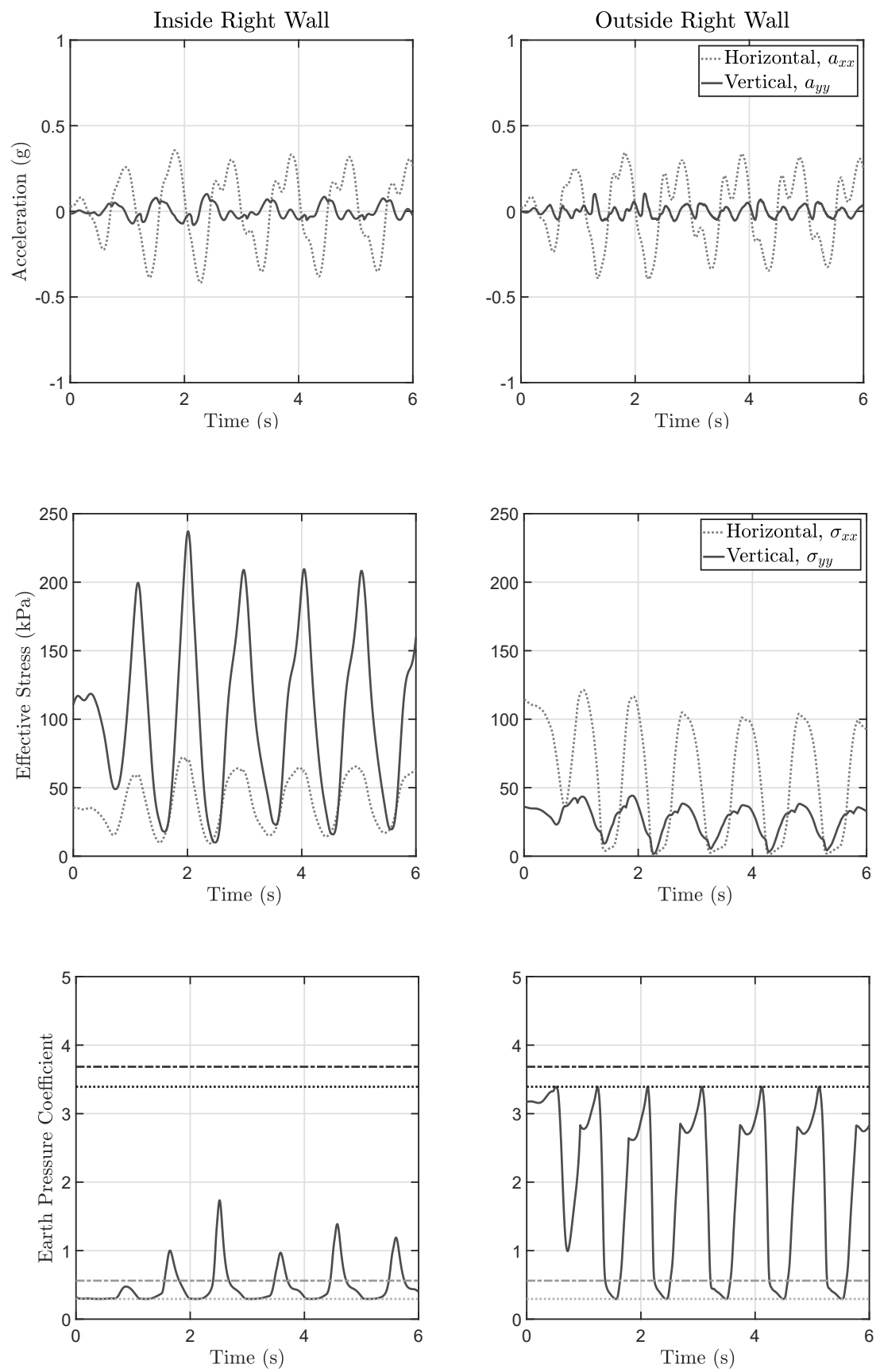

\begin{tabular}{|ll|}
\hline -K Mobilised, $\frac{\sigma_{x x}}{\sigma_{y y}}$ & $\cdots \cdot$ Active Limit, $K_{A}$ \\
$\cdots \cdot$ Passive Limit, $K_{P}$ & ---MO Active Limit, $+0.35 g, K_{A D}$ \\
---MO Passive Limit, $+0.35 g, K_{P D}$ & \\
\hline
\end{tabular}

(b) Horizontal and vertical response of the soil. Vertical accelerations can only partly justify the vertical stress oscillations, which are predominantly due to the rocking soil mass. The dynamic earth pressure coefficient mobilised, accounting for the dynamic vertical stresses, can violate pseudo static coefficients but are always bounded by the Rankine coefficients

Figure 13: Dynamic response of the soil around the right wall 
depend on the ability of the wall-soil interface to supply shear stresses. There is a dynamic redistribution of vertical soil stresses needed to equilibrate the rocking soil mass at every elevation. A secondary effect is the vertical accelerations from compatible wall-soil displacements altering the vertical stresses with respect to the geostatic distributions.

Tracking the ratios of the horizontal to vertical stresses suggests a simpler interpretation of the dynamic stress states that can be generated around a buried or embedded soil-structure system. For the analyses and loading magnitudes in this paper, the critical state friction angle could be solely used to define the active and passive limit states the dynamic soil response was bounded by. Dynamic earth pressure coefficients, as traditionally defined, may obfuscate the soil behaviour as neither the horizontal or vertical stress distributions need be linear or constant with time. It is instead recommended that efforts to better understand and design for the dynamic response of soil-structure systems should enforce horizontal stresses that are bounded by the expected or predicted dynamic vertical stresses multiplied with earth pressure coefficients calculated based on the appropriate frictional strength of the material.

\section{References}

Azeiteiro, R. J. N., Coelho, P. A. L. F., Taborda, D. M. G. and Grazina, J. C. D. (2017). Critical State Based Interpretation of the Monotonic Behavior of Hostun Sand, Journal of Geotechnical and Geoenvironmental Engineering 143(5): 1-14.

Burki, N. K. and Richards, R. J. (1975). Photoelastic analysis of a cofferdam, Journal of Geotechnical and Geoenvironmental Engineering 101(ASCE \# 11135 Proc. Paper).

Campbell, D. J., Cheney, J. A. and Kutter, B. L. (1991). Boundary effects in dynamic centrifuge model tests, Centrifuge 1991 : proceedings of the International Conference Centrifuge 1991, A.A. Balkema, Boulder, Colorado, pp. 441-448.

Chan, A. H. C. (1988). A unified Finite Element Solution to Static and Dynamic problems of Geomechanics, $\mathrm{PhD}$ thesis, University College of Swansea.

Chang, M. F. and Chen, W. F. (1990). Chapter 6 - Some Practical Considerations in Design of Rigid Retaining Structures, in W. F. CHEN and X. L. LIU (eds), Development in Geotechnical Engineering, Vol. 52 of Developments in Geotechnical Engineering, Elsevier, pp. 231-308.

Chopra, A. K. (2012). Dynamics of structures : theory and applications to earthquake engineering, Prentice Hall.

Choudhury, D. and Nimbalkar, S. S. (2006). Seismic passive resistance by pseudo-dynamic method, Géotechnique 56(7): 517-520.

Clough, G. W. and Duncan, J. M. (1971). Finite Element Analyses of Retaining Wall Behavior, Journal of the Soil Mechanics and Foundations Division, 97(12).

Coe, C. J., Prevost, J. H. and Scanlan, R. H. (1985). Dynamic stress wave reflections/attenuation: Earthquake simulation in centrifuge soil models, Earthquake Engineering \& Structural Dynamics 13(1): 109-128.

Cook, R., David, S., Michael, E. and Robert, J. (2002). Concepts and applications of finite element analysis, 4th edn, Wiley.

Coulomb, C. A. (1776). An attempt to apply the rules of maxima and minima to several problems of stability related to architecture, Mémoires de l'Académie Royale des Sciences 7: 343-382.

Dashti, S., Gillis, K. and Ghayoomi, M. (2012). Sensing of Lateral Seismic Earth Pressures in Geotechnical Centrifuge Models, 15th World Conference on Earthquake Engineering, Lisbon Portugal, Sociedade Portuguesa de Engenharia Sismica (SPES), pp. 1-10.

Desai, C. S., Drumm, E. C. and Zaman, M. (1985). Cyclic Testing and Modelling of Interfaces, Journal of Geotechnical Engineering - ASCE I(6): 793-815. 
Dubrova, G. A. (1963). Interaction of soil and structures, Izd. Rechnoy Transport, Moscow .

Ebeling, R. M. and Morrison, E. E. J. (1992). The Seismic Design of Waterfront Retaining Structures, Technical report.

Hinton, E. E. and Owen, D. R. J. (1979). An introduction to finite element computations, Pineridge Press.

Katona, M. C. and Zienkiewicz, O. C. (1985). A unified set of single step algorithms part 3: The beta$\mathrm{m}$ method, a generalization of the Newmark scheme, International Journal for Numerical Methods in Engineering 21(7): 1345-1359.

Khosravi, M. H., Pipatpongsa, T. and Takemura, J. (2016). Theoretical analysis of earth pressure against rigid retaining walls under translation mode, Soils and Foundations 56(4): 664-675.

Kitajima, S., Noda, S. and Nakayama, T. (1981). An Experimental Study on the Static Stability of the Steel Plate Cellular Bulkhead with Embedment, Technical report.

Kontoe, S., Zdravković, L., Potts, D. M. and Menkiti, C. O. (2011). On the relative merits of simple and advanced constitutive models in dynamic analysis of tunnels, Geotechnique 61(10): 815-829.

Kramer, S. L. (1996). Geotechnical Earthquake Engineering, Prentice Hall.

Madabhushi, S. P. G., Houghton, N. E. and Haigh, S. K. (2006). A new automatic sand pourer for model preparation at University of Cambridge, Proceedings of the Sixth International Conference on Physical Modelling in Geotechnics, Taylor \& Francis, Hong Kong, pp. 217-222.

Madabhushi, S. S. C. (2018). Multi-Hazard Modelling of Dual Row Retaining Walls, Phd thesis, University of Cambridge.

Madabhushi, S. S. C. and Haigh, S. K. (2018a). A Comparison between the Dynamic Behaviour of Flexible Dual Row Walls Founded in Dry and Liquefiable Sands, 1st International Conference on Press-in Engineering, pp. 265-272.

Madabhushi, S. S. C. and Haigh, S. K. (2018b). Combining Experimental and Numerical Methods to investigate the seismic performance of dual row retaining walls in Dry Sand, 16th European Conference on Earthquake Engineering.

Madabhushi, S. S. C. and Haigh, S. K. (2018c). Measuring the behaviour of dual row retaining walls in dry sands using centrifuge tests, International Conference of Physical Modelling in Geotechnics, London, UK, pp. 639-644.

Madabhushi, S. S. C. and Haigh, S. K. (2018d). Using Tactile Pressure Sensors to Measure Dynamic Earth Pressures around Dual Row Walls, International Journal of Physical Modelling in Geotechnics 19(2): 5871.

Madabhushi, S. S. C. and Haigh, S. K. (2019). Centrifuge Testing of Dual Row Walls in Dry Sand: the influence of Earthquake sequence and multiple flights, Soil Dynamics and Earthquake Engineering 125.

Michalowski, R. L. and Park, N. (2003). Arching in Granular Soils, The First Japan - U.S. Workshop on Testing, Modeling, and Simulation (143): 255-268.

Mononobe, N. and Matsuo, H. (1929). On determination of earth pressure during earthquake, Proceedings of the World Engineering Congress, Tokyo, Vol. 9, Kogakkai, Tokyo, Japan, p. 176.

Morrison, C. S. (1995). The development of a modular finite element program for analyses of soil-structure interaction, PhD thesis, Virginia Polytechnic Institute and State University.

Okabe, S. (1924). General Theory on Earth Pressure and Seismic Stability of Retaining Wall and Dam, Journal of Japan Society of Civil Engineers 10(6): 1277-1323. 
Paik, K. H. and Salgado, R. (2003). Estimation of active earth pressure against rigid retaining walls considering arching effects, Géotechnique 53(7): 643-653.

Paikowsky, S. G. and Hajduk, E. L. (1997). Calibration and Use of Grid-Based Tactile Pressure Sensors in Granular Material, Geotechnical Testing Journal 20(2): 218-241.

Rankine, M. W. J. (1856). On the Stability of Loose Earth, Philosophical Transactions of the Royal Society of London 147: 9-27.

Schofield, A. N. (1980). Cambridge Geotechnical Centrifuge Operations, Géotechnique 30(3): 227-268.

Scott, R. F. (1973). Earthquake-induced pressures on retaining walls, Proceedings of the 5th World Conference on Earthquake Engineering, Vol. 2, Rome, Italy, pp. 1611-1619.

Semblat, J. F., Brioist, J. J. and Luong, M. P. (1999). Mean stress dependent damping of seismic waves in sand, Earthquake Geotechnical Engineering pp. 95-100.

Steedman, R. S. and Zeng, X. (1990). The influence of phase on the calculation of pseudo-static earth pressure on a retaining wall, Géotechnique 40(1): 103-112.

Teymur, B. and Madabhushi, S. P. G. (2003). Experimental study of boundary effects in dynamic centrifuge modelling, Géotechnique 53(7): 655-663.

White, D. J., Take, W. A. and Bolton, M. D. (2003). Soil deformation measurement using particle image velocimetry (PIV) and photogrammetry, Géotechnique 53(7): 619-631.

Wood, J. H. (1975). Earthquake-induced Pressures on a Rigid Wall Structure, Bulletin of the New Zealand Society for Earthquake Engineering 8(3): 175-186.

Zienkiewicz, O. C., Chan, A. H. C., Pastor, M., Schrefler, B. A. and Shiomi, T. (2001). Computational Geomechanics with Special Reference to Earthquake Engineering, Vol. 25, Wiley. 